\title{
On the Uptake of Materials by the Intact Liver
}

\author{
THE TRANSPORT AND NET REMOVAL OF GALACTOSE
}

\author{
Carl A. Goresky, Glen G. Bach, and Brita E. Nadeau \\ From the McGill University Medical Clinic in the Montreal General Hospital, \\ and the Departments of Medicine and of Mechanical Engineering, \\ McGill University, Montreal, Quebec, Canada
}

A в S T R A C T D-galactose, a monosaccharide rapidly phosphorylated within liver cells, is irreversibly removed from the portal circulation. We have studied the kinetic relations between the hepatic cell entry process and the metabolic sequestration process, by means of the multiple indicator dilution technique. Labeled red blood cells (a vascular indicator), labeled sucrose (an extracellular reference), and labeled galactose were rapidly injected into the portal vein, and from rapidly sampled hepatic venous blood, normalized outflow-time patterns were secured. The labeled red cell curve rises to the highest and earliest peak, and decays rapidly; and that for labeled sucrose rises to a later and lower peak. Its extrapolated recovery is equivalent to that of the labeled red cells. At low blood galactose concentrations, the labeled galactose appears at the outflow with labeled sucrose, but is much reduced in magnitude, and exhibits a long tailing. Its outflow recovery is much reduced. At high blood galactose concentrations, the initial part of the profile increases towards that for labeled sucrose, the tailing becomes much larger in magnitude, and the outflow recovery becomes virtually complete.

We have modeled the uptake of labeled galactose, and find two parts to the predicted outflow pattern, corresponding to our experimental observations: throughput material, which sweeps past the cell surface in the extracellular space; and returning material, which has entered the cells but escaped the sequestration process. Analysis of the data by use of this model provides estimates of both transmembrane fluxes and rates of sequestration. The capacity of the process subserving cell entry is found to be 40 times that for phosphorylation; and, whereas the $K_{m}$ value for sequestration is less than $15 \mathrm{mg} / 100 \mathrm{ml}$, that for entry is approximately $500 \mathrm{mg} / 100 \mathrm{ml}$. Both processes are relatively stereo-

Received for publication 3 February 1971 and in revised form 29 December 1972. specific: the entry of the L-stereoisomer is very slow and it undergoes no significant amount of metabolic sequestration. The sequestration process produces a lobular intracellular concentration gradient; and this gradient, in turn, prduces some uncertainty in the estimate of the true $K_{m}$ value for the sequestration process.

\section{INTRODUCTION}

D-galactose is a hexose which is removed in a net fashion from the circulation by the liver. When the plasma level of galactose is high, the removal rate is constant, and so long as the hepatic blood flow is constant, a constant arterial-hepatic vein difference results; and when the plasma concentration is low (less than $30 \mathrm{mg} / 100 \mathrm{ml}$ ), the extraction is almost complete, i.e., the hepatic venous blood is almost completely cleared of galactose. The extraction of galactose in the lower range is so large (an average of $88 \%$ in normal man) that its constant infusion has been used as a basis for the estimation of hepatic blood flow $(1,2)$. Underlying these observations are two sets of phenomena: the process of membrane carrier transport subserving the entry of galactose into and its exit from liver cells; and a process of phosphorylation, of conversion via galactokinase, into galactose-1-phosphate, a compound which does not freely pass through cell membranes. This process effectively sequesters and removes galactose from any free intracellular pool. This compound, in turn, is ordinarily rapidly converted to glucose-1-phosphate by the enzyme galactose-1-phosphate uridyl transferase (the enzyme usually deficient in galactosemia $(3,4))$; and this product is metabolized, incorporated into glycogen, or released as free intracellular glucose.

It is our purpose in this paper to explore experimentally the relations between the hepatic membrane transport and intracellular disposal processes for galactose; 


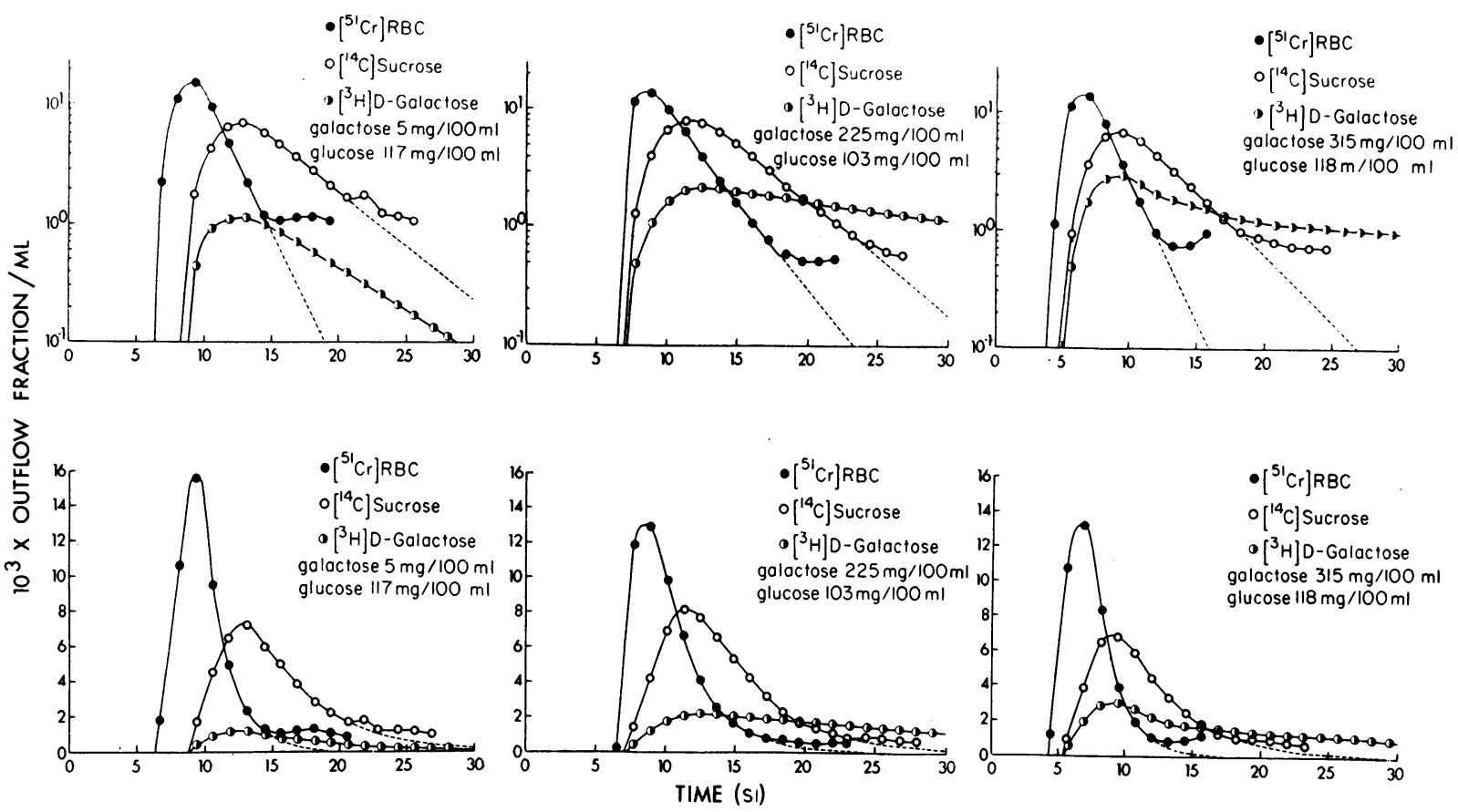

FIGURE 1 Change in the outflow profile for labeled galactose with change in the blood galactose levels. Abscissas: time in seconds. Ordinates: outflow fraction per milliliter. The scale is logarithmic in the upper panel, and linear in the lower. The time delay in the collecting system was $2.54,2.48$, and $2.52 \mathrm{~s}$, respectively, in these three experiments.

to use the experimental observations as a guide to the formulation of a model designed to describe the kinetic interactions between the transport of galactose and its intracellular sequestration; and finally to analyze the general implications of the kind of modeling which results from the study of these specific processes. The modeling which we employ is an extension of the general transport model (with no intracellular removal process) which we developed to describe the uptake of ${ }^{86} \mathrm{Rb}$ by the intact liver (5).

\section{METHODS}

We use as our experimental approach the rapid single injection multiple indicator dilution technique (6). We inject three substances simultaneously: ${ }^{51} \mathrm{Cr}$-labeled red cells, as a vascular reference substance; ${ }^{14} \mathrm{C}$-labeled sucrose, as a reference extracellular material, which undergoes flow-limited distribution into the extracellular space, but does not enter liver cells to a significant extent during a single passage (7) ; and ${ }^{3} \mathrm{H}$-labeled galactose, the substance which we wish to examine. In each instance the hematocrit of the injection mixture is adjusted so that it is equal to that of the portal venous blood. The mixture is injected as rapidly as possible into a portal venous catheter. The experiments are carried out in anesthetized dogs and the operative procedures and the procedures for the preparation and analysis of samples are those described previously $(5,7)$. Galactose equilibrates in red cell water (8) but in the dog isotopic equilibration requires about $4 \mathrm{~h}$. To avoid the carriage of labeled galactose by the red cells, the injection mixture was constituted only at the time of the run.
Several other maneuvers were carried out in an attempt to more precisely characterize the mechanisms involved. The D-galactose level was increased by steady infusion, so that the characteristics of the saturation phenomena could be defined. Both glucose infusion and phlorizin infusion were used to demonstrate competitive phenomena; and the transport of $\beta$-methyl D-galactoside and of $\beta$-methyl D-thiogalactoside, and of $\mathrm{L}$-galactose were studied, in order to demonstrate the effects of minor and major changes in molecular architecture.

Special materials. The following special materials were used: $\left[{ }^{51} \mathrm{Cr}_{2}\right] \mathrm{Na}_{2} \mathrm{O}_{7}$ solution, $6 \mathrm{Ci} / \mathrm{mmol} ;{ }^{1}\left[6{ }^{3} \mathrm{H}\right] \mathrm{D}$-galactose, $6.9 \mathrm{Ci} / \mathrm{mmol}^{2}$ or $\left[1-{ }^{3} \mathrm{H}\right] \mathrm{D}$-galactose, $0.2 \mathrm{Ci} / \mathrm{mmol} ;{ }^{2}$ [U-1 $\left.{ }^{14} \mathrm{C}\right]-$ sucrose, $4.7 \mathrm{mCi} / \mathrm{mmol} ;{ }^{2}[\beta$-methyl-14 $\mathrm{C}] \mathrm{D}$-galactoside, 7.6 $\mathrm{mCi} / \mathrm{mmol} ;{ }^{2}\left[\beta-\right.$ methyl- $\left.{ }^{14} \mathrm{C}\right] \mathrm{D}$-thiogalactoside, $7.7 \mathrm{mCi} / \mathrm{m}$ $\mathrm{mol} ;{ }^{2}$ and $\left[1-{ }^{3} \mathrm{H}\right] \mathrm{L}$-galactose, $1.6 \mathrm{Ci} / \mathrm{mmol}^{3}$

\section{RESULTS}

In order to relate the outflow pattern of one substance to that of another, they are each expressed as a fraction of the amount injected per milliliter vs. time. Fig. 1 illustrates the relative change in the form and magnitude of the outflow profile for labeled galactose with change in the serum concentration of galactose. In each panel we find the relation expected between the labeled sucrose and labeled red cell curves, as a result of the extravascular flow-limited distribution of sucrose (7):

\footnotetext{
${ }^{1}$ Charles E. Frosst and Co., Montreal, Quebec, Canada.

2 New England Nuclear Corp., Boston, Mass.

${ }^{3}$ Volk Radiochemical Co., Burbank, Calif.
} 
the values for the labeled sucrose are lower on the upslope; the peak for the curve is lower and is delayed so that it falls beyond the downslope of the labeled red cell curve; the downslope decays more slowly; and recirculation appears later. The labeled red cell and sucrose curves are corrected for recirculation by linear extrapolation on the semilogarithmic plot, in the usual manner (9). When no galactose has been infused (the left hand panel), the galactose curve consists of a low-in-magnitude early peak, "included" under the sucrose curve, followed by an abbreviated tailing. The galactose tailing does not reach sufficient magnitude to cross the labeled sucrose curve. In the middle and right-hand panels the effects of progressive increase in the serum galactose level are illustrated. In the middle panel the early part of the curve has increased in magnitude and this now blends with a later relatively pronounced tailing, which crosses the primary downslope of the labeled sucrose curve. In the experiment illustrated in the right-hand panel, where the serum galactose is quite high, the curve itself appears to be resolving into two separate parts: a first distinguishable larger peak, contained within the labeled sucrose curve; and a second prolonged component, a later low-in-magnitude tailing which crosses the primary downslope of the sucrose curve.

Those parameters which can be obtained directly from the study are assembled in Table I. These include values for flow, for hepatic perfusion (i.e., for flow per unit weight of tissue), and for the mean transit times for labeled red cells and labeled sucrose. The outflow recoveries of labeled red cells and labeled sucrose are equivalent (the ratio of the area under the labeled sucrose curve to that under the curve for labeled red cells is $1.016 \pm 0.045[\mathrm{SD}])$. The manner in which the primary galactose curve may be separated from recirculating label, so that we may obtain recoveries and mean transit times, is not apparent, at this point in the development. It is obvious, however, that the outflow recovery of labeled galactose will be grossly reduced at the lowest serum galactose values.

The discussion of the change in form of the labeled galactose curves after glucose loading and after phlorizin infusion, and the form of the $\beta$-methyl galactoside curves is best deferred until we have presented an analysis of the effects of galactose loading.

The use of modeling to analyze the dilution experiments. In order to provide a basis for the interpretation of the results of these experiments, we have developed in the Appendix a model of the kinetic processes involved, the flow-limited distribution of galactose out to the cell membrane, its passage across that membrane, and its intracellular sequestration. The outflow profile is again found to consist of two parts: throughput and
TABle I

Transit Time and Flow Data from the Dilution Curies

\begin{tabular}{|c|c|c|c|c|c|c|}
\hline $\begin{array}{c}\text { Exp. } \\
\text { no. }\end{array}$ & $\begin{array}{l}\text { Body } \\
\text { wt }\end{array}$ & $\begin{array}{l}\text { Liver } \\
\text { wt }\end{array}$ & $\begin{array}{c}\text { Hepatic } \\
\text { perfusion }\end{array}$ & $\overline{\mathrm{t}}_{\mathrm{RBC}} *$ & $\overline{\mathrm{t}}_{\text {suer }} *$ & $\begin{array}{l}\text { Sucrose } \\
\text { extra- } \\
\text { vascular } \\
\text { space }\end{array}$ \\
\hline & $\mathrm{kg}$ & $g$ & $\begin{array}{c}m l \cdot s^{-1} \\
\cdot g^{-1}\end{array}$ & $s$ & $s$ & $\begin{array}{c}(m l \text { plasma }) \\
\cdot g^{-1}\end{array}$ \\
\hline \multicolumn{7}{|c|}{ D-galactose experiments } \\
\hline 1 & 13 & 356 & 0.019 & 8.13 & 14.91 & 0.076 \\
\hline 2 & 16 & 390 & 0.043 & 7.52 & 13.00 & 0.148 \\
\hline $3 \S$ & 16 & 359 & 0.032 & 5.92 & 10.06 & 0.086 \\
\hline $4 \S$ & 15 & 319 & 0.055 & 5.60 & 8.92 & 0.115 \\
\hline $5 \S$ & 16 & 480 & 0.030 & 7.72 & 11.23 & 0.113 \\
\hline 68 & 18 & 411 & 0.050 & 4.61 & 9.12 & 0.142 \\
\hline $7 \S$ & 18 & 497 & 0.046 & 6.05 & 10.55 & 0.149 \\
\hline $8 \S$ & 17 & 452 & 0.024 & 8.57 & 16.67 & 0.137 \\
\hline $9 \S$ & 14 & 460 & 0.027 & 10.68 & 14.87 & 0.087 \\
\hline $10 \S$ & 14 & 411 & 0.047 & 4.97 & 9.01 & 0.140 \\
\hline \multicolumn{7}{|c|}{ D-glucose loading } \\
\hline $11 \|$ & 16 & 507 & 0.071 & 4.06 & 6.87 & 0.120 \\
\hline $12 \|$ & 14 & 414 & 0.031 & 5.54 & 9.66 & 0.089 \\
\hline \multicolumn{7}{|c|}{ Phlorizin infusion } \\
\hline 13 | & 17 & 450 & 0.024 & 7.63 & 13.23 & 0.074 \\
\hline \multicolumn{7}{|c|}{ L-galactose experiments } \\
\hline 14 & 15 & 389 & 0.035 & 7.94 & 13.40 & 0.130 \\
\hline 15 & 15 & 459 & 0.025 & 6.36 & 12.26 & 0.100 \\
\hline $16 \S$ & 18 & 468 & 0.044 & 4.82 & 8.98 & 0.113 \\
\hline
\end{tabular}

* The mean transit times were corrected for the catheter transit times. $\ddagger$ The sucrose extravascular space was calculated as the product of the plasma flow and the difference between labeled sucrose and red cell mean transit times.

$\S \mathrm{D}$-galactose was infused for the $40 \mathrm{~min}$ before and during the run, in these experiments.

II D-glucose was infused for $30 \mathrm{~min}$ before and during the run.

I Phlorizin $(0.24 \mathrm{~g} / \mathrm{kg})$ was infused into the portal vein for the $30 \mathrm{~min}$ before and during the run. Systemic hypotension was avoided.

returning material (which has entered the cells and returned to the sinusoids). It differs from the case in which there is transport but no sequestration (5) in the presence of a damping function, which progressively decreases the magnitude of the outflow returning material with time. This last effect is best appreciated by referring to Fig. 10, a set of computed outflow profiles, in the Appendix.

Using this model, we have derived from the interrelation between the experimental curves for the three substances (vascular reference, extracellular reference, and the material both transported and sequestered within cells) numerical estimates of five parameters: $p \gamma$ (the extracellular space ratio), to (the large vessel transit time), $k_{1} \theta /[1+p \gamma]$ (the cellular influx rate constant, multiplied by the ratio of the cellular space to the total space, vascular + extracellular, outside cells), $k_{2} / r$ (the cellular efflux rate constant, divided by the partition coefficient for galactose in cells), and $k_{3} / r$ (the sequestration constant, divided by the partition coefficient for galactose in cells). Initial graphical estimates (7) of $p \gamma$ and $t_{0}$ are obtained from the two reference curves, on 


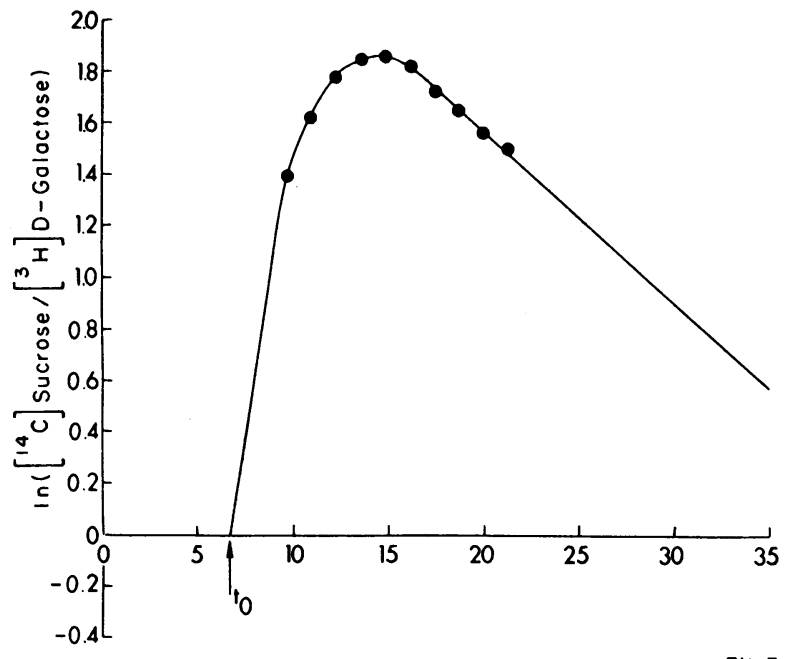

$\operatorname{TIME}(s)$

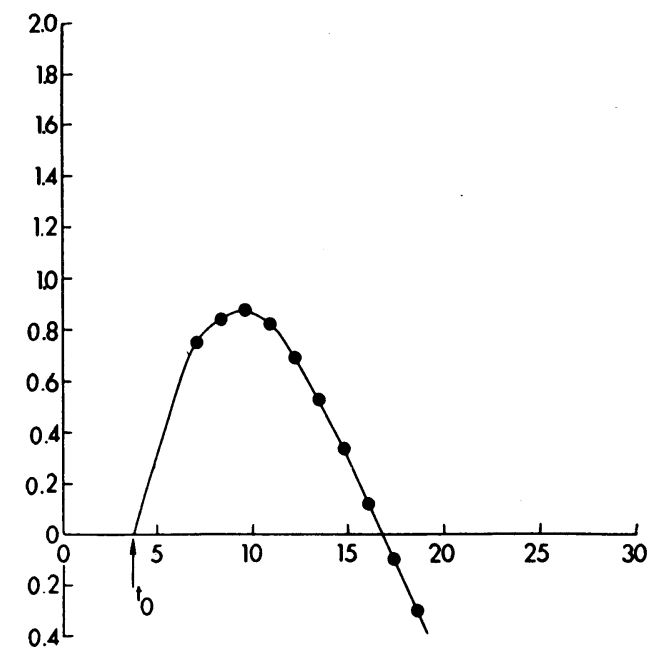

FIGURE 2 Plot of the natural logarithm of the ratio (sucrose outflow fraction per milliliter/ galactose outflow fraction per milliliter) vs. time. The panel on the left corresponds to the left-hand panel of Fig. 1 (galactose level $5 \mathrm{mg} / 100 \mathrm{ml}$ ); and that on the right, to the righthand panel of Fig. 1 (galactose level $315 \mathrm{mg} / 100 \mathrm{ml}$ ). Rough initial estimates of the parameter $k_{1} \theta /(1+p \gamma)$ are obtained from the slope of the line joining the $t_{0}$ value to the first point. The slope of the line is less in the second panel, where galactose has been infused.

the basis of the relationship described by equation $11 \mathrm{~B}$. These are then optimized on the computer by minimizing the sum of the squares of the differences between a labeled red cell curve and a labeled sucrose curve, which has been scaled in time and magnitude in the manner appropriate to make it superimpose upon the red cell curve (5). For this group of experiments, the average relative coefficient of variation for this fitting procedure was 0.082 . Since the data were obtained by means of a continuous sampling system, each sample was considered to have been integrated over its collection interval.

Next we need a first approximation to the parameter $k_{1} \theta /(1+p \gamma)$. We expect that the initial samples containing labeled galactose will be composed chiefly of throughput material, and hence (from equations $11 \mathrm{~B}$ and $11 \mathrm{C}$ of the Appendix) that the initial slope of a plot of the natural logarithm of the ratio of the labeled sucrose to labeled galactose outflow fraction per milliliter vs. time will provide this rough estimate. Plots of the first and third experiments of Fig. 1, for which blood galactose levels are low and high, respectively, are illustrated in Fig. 2. For the experiment with the higher galactose level, the initial slope (the approximation to $k_{1} \theta /[+p \gamma]$ ) is less than it is for the experiment with the lower galactose level. For the experiment with the low galactose level, the locus on the plot reaches a relatively higher peak and then begins to drop; whereas for the experiment with the high galactose level, a much lower peak is followed by a downslope which crosses the zero ordinate value at the time when the galactose outflow fraction per milliliter crosses the sucrose curve.

In addition we need rough initial estimates of the parameters $k_{2} / r$ and $k_{3} / r$. If we suppose that the galactose transport system is equilibrative (i.e. $k_{1}=k_{2}$ ), the ratio $\left[k_{1} \theta /(1+p \gamma)\right] /\left[k_{2} / r\right]$ would be expected to have a value corresponding to $r \theta /(1+p \gamma)$, the ratio of the cellular space to the total space, vascular + extracellular, outside cells. If the extracellular sucrose space is of the order of $10 \%$ of liver weight (7), and the sinusoidal plasma space is of the same order of magnitude, this ratio would be expected to have a value of the order of 3.0. Initial values of $k_{2} / r$ were therefore estimated to be one-third of the initial values for $k_{1} \theta /(1+p \gamma)$; and the initial values for $k_{3} / r$ were approximated by extrapolating from the data of Tygstrup and Winkler (1).

A computed labeled galactose curve was then generated from the labeled sucrose curve, on the basis of the theoretical development outlined in the Appendix, and optimal values for $k_{1} \theta /(1+p \gamma), k_{2} / r$, and $k_{3} / r$ were obtained by minimizing the sum of the squares of the differences between the experimental and computed curves. We have used here, as previously (5), the whole of the reliable experimental information (upslope, peak, and early downslope) to calculate parameters. The results of the fitting procedure for the experiments illustrated in the first and third panels of Fig. 1 are illustrated in Figs. 3 and 4. In each figure the galactose curve has been resolved into two components: throughput, and exchanging material (which 

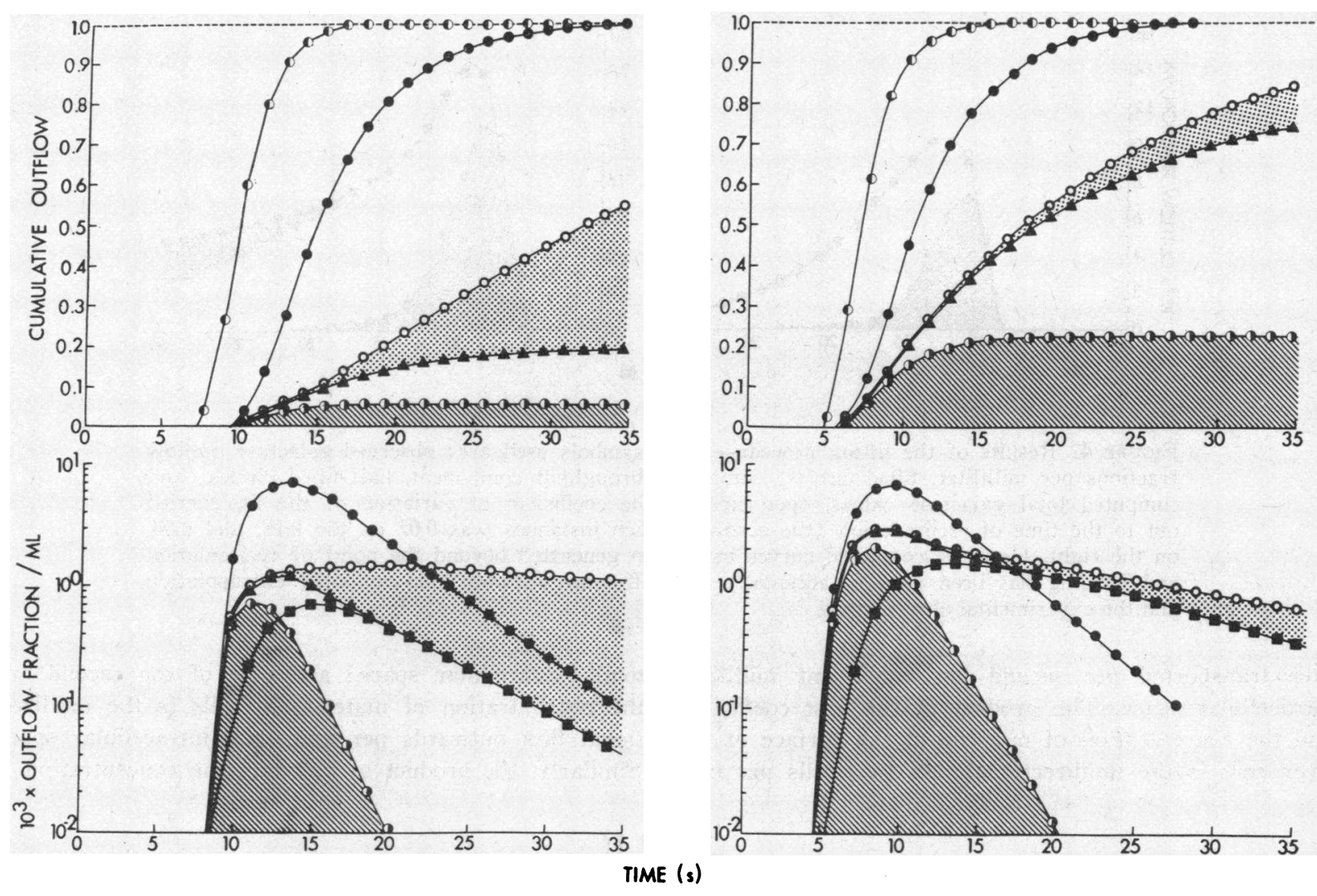

Figure 3 Resolution of the components of the galactose curve. The cumulative outflow is illustrated in the upper panels; and the observed outflow fractions/milliliter, in the lower panels. The panel on the left corresponds to the left-hand panel of Fig. 1 (galactose level $5 \mathrm{mg} / 100 \mathrm{ml}$ ); and that on the right, to the right-hand panel of Fig. 1 (galactose level 315 $\mathrm{mg} / 100 \mathrm{ml}$ ). The symbols used are: labeled red cells, left half-filled circles; labeled sucrose, solid circles; labeled galactose, filled triangles; resolved first components of the galactose, right half-filled circles; resolved second component of the galactose, filled squares; and the form the galactose curve would have had if none of this substance had been sequestered, if all of it had returned to the circulation, open circles. The resolved first components of the galactose curves have been shaded, to accentuate their relation to the labeled sucrose curves; and the effect produced by the parameter $k_{3} / r$, in each panel, has been demonstrated by shading the area between the galactose curve and the curve expected for galactose if $k_{3} / r$ had been zero. In order to avoid a confusion of points, only the fitted curves and their resolved components were displayed in this illustration.

has entered the cells and escaped the sequestering process, so that it emerges at the outflow). In Fig. 3 the form the curve would have had if no sequestration had occurred is also displayed. Both figures demonstrate that, at the higher galactose level, proportionately more of the labeled galactose emerges as throughput; and the proportion which is sequestered is greatly diminished. Fig. 4 illustrates the relative concordance between the observed and computed galactose curves, when the fit was carried out by use of all the reliable experimental information, that part up to but excluding apparent recirculation. For the whole group of experiments, the average relative coefficient of variation of the fit was 0.045 . Beyond the point of recirculation, the computed extrapolation of the fitted galactose curve deviates very largely from the experimental data, for the case with high galactose concentration. In most of the experiments with the higher galactose concentrations, linear extrapolation of the original galactose data on the semilogarithmic plot would have resulted in inordinately large areas under the galactose curve.

The best fit parameters are aggregated in Table II.

Changes in the derived parameters with substrate concentration. The transport coefficients $k_{1} \theta /(1+p \gamma)$ and $k_{2} / r$ have the dimensions $\mathrm{ml} \cdot \mathrm{s}^{-1} \cdot \mathrm{ml}^{-1}$. For the first, these are milliliter plasma transported per second per equivalent milliliter total space, vascular + extracellular, outside the cells; and, for the second, equivalent milli- 


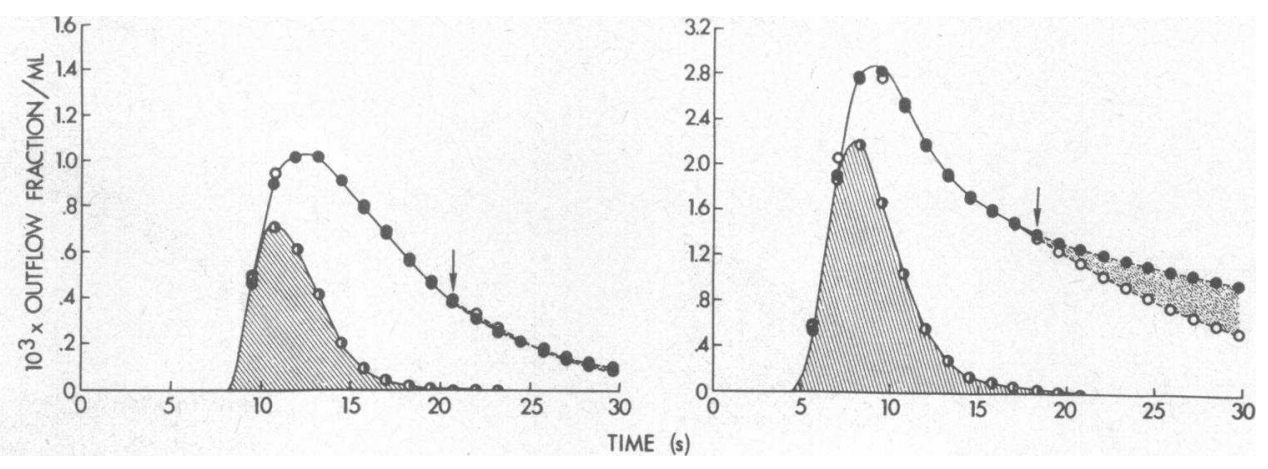

Figure 4 Results of the fitting procedure. The symbols used are: observed galactose outflow fractions per milliliter, filled circles; computed throughput component, half-filled circles; and computed total galactose values, open circles. The coefficient of variation of the fit, carried out to the time of recirculation (the arrow, in each instance) was 0.07 on the left; and 0.08 , on the right. Here the computed curves have been generated beyond the point of recirculation and shading has been used to accentuate the difference between the computed extrapolation and the experimental observations.

liter transported per second per equivalent milliliter intracellular space. The product of the first coefficient and the concentration of material at the surface of the liver cells is the unidirectional flux into cells per unit total extracellular space; and that of the second and the concentration of material in cells is the unidirectional flux outwards per unit total intracellular space. Similarly, the product of $k_{3} / r$ and the concentration of

TABLE II

Glucose and Galactose Concentrations and Derived Parameters

\begin{tabular}{|c|c|c|c|c|c|c|c|c|c|c|c|}
\hline \multirow{2}{*}{$\begin{array}{c}\text { Exp } \\
\text { no. }\end{array}$} & \multirow{2}{*}{$\begin{array}{l}\text { Blood } \\
\text { galactose } \\
\text { concen- } \\
\text { tration }\end{array}$} & \multirow{2}{*}{$\begin{array}{l}\text { Plasma } \\
\text { glucose } \\
\text { concen- } \\
\text { tration }\end{array}$} & \multirow[b]{2}{*}{ Hct } & \multicolumn{4}{|c|}{ Original curves } & \multicolumn{4}{|c|}{ Corrected curves* } \\
\hline & & & & $p \gamma$ & $k_{1} \theta /(1+p \gamma)$ & $k_{2} / r$ & $k_{3} / r$ & $p \gamma$ & $k_{1} \theta /(1+p \gamma)$ & $k_{2} / r$ & $k_{3} / r$ \\
\hline & \multicolumn{3}{|c|}{$\mathrm{mg} / 100 \mathrm{ml} \mathrm{mg} / 100 \mathrm{ml}$} & & $s^{-1}$ & $s^{-1}$ & $s^{-1}$ & & $s^{-1}$ & $s^{-1}$ & $s^{-1}$ \\
\hline \multicolumn{12}{|c|}{ D-galactose experiments } \\
\hline 1 & 5 & 106 & 0.42 & 1.18 & 0.398 & 0.172 & 0.222 & 1.28 & 0.387 & 0.141 & 0.196 \\
\hline 2 & 5 & 117 & 0.37 & 1.34 & 0.404 & 0.187 & 0.188 & 1.44 & 0.399 & 0.180 & 0.190 \\
\hline 3 & 104 & 89 & 0.35 & 0.99 & 0.390 & 0.248 & 0.048 & 1.17 & 0.409 & 0.218 & 0.036 \\
\hline 4 & 199 & 79 & 0.37 & 0.68 & 0.231 & 0.205 & 0.017 & 0.83 & 0.246 & 0.191 & 0.009 \\
\hline 5 & 225 & 103 & 0.29 & 0.79 & 0.371 & 0.236 & 0.022 & 1.03 & 0.288 & 0.184 & 0.013 \\
\hline 6 & 226 & 85 & 0.37 & 1.33 & 0.299 & 0.247 & 0.059 & 1.87 & 0.340 & 0.225 & 0.040 \\
\hline 7 & 240 & 88 & 0.28 & 0.85 & 0.279 & 0.219 & 0.021 & 1.07 & 0.290 & 0.210 & 0.017 \\
\hline $\begin{array}{l}8 \\
9\end{array}$ & $\begin{array}{l}273 \\
282\end{array}$ & $\begin{array}{r}74 \\
165\end{array}$ & $\begin{array}{l}0.30 \\
0.24\end{array}$ & $\begin{array}{l}1.57 \\
0.48\end{array}$ & $\begin{array}{l}0.290 \\
0.162\end{array}$ & $\begin{array}{l}0.137 \\
0.112\end{array}$ & $\begin{array}{l}0.019 \\
0.027\end{array}$ & $\begin{array}{l}1.68 \\
0.62\end{array}$ & $\begin{array}{l}0.299 \\
0.161\end{array}$ & $\begin{array}{l}0.127 \\
0.108\end{array}$ & $\begin{array}{l}0.013 \\
0.025\end{array}$ \\
\hline 10 & 315 & 118 & 0.26 & 1.13 & 0.257 & 0.207 & 0.023 & 1.26 & 0.261 & 0.200 & 0.023 \\
\hline \multicolumn{12}{|c|}{ Glucose loading } \\
\hline 11 & 4 & 602 & 0.40 & 0.90 & 0.240 & 0.083 & 0.026 & 1.03 & 0.273 & 0.095 & 0.045 \\
\hline 12 & 4 & 1511 & 0.30 & 0.93 & 0.176 & 0.083 & 0.054 & 2.04 & 0.214 & 0.092 & 0.052 \\
\hline \multicolumn{12}{|c|}{ Phlorhizin infusion } \\
\hline 13 & 8 & 132 & 0.32 & 1.02 & 0.079 & 0.020 & 0.062 & 1.31 & 0.082 & 0.021 & 0.063 \\
\hline \multicolumn{12}{|c|}{ L-galactose experiments } \\
\hline 14 & 8 & 80 & 0.32 & 0.80 & 0.048 & 0.058 & 0.013 & 1.00 & 0.052 & 0.062 & 0.012 \\
\hline 15 & 10 & 101 & 0.33 & 1.29 & 0.074 & 0.026 & 0.009 & 1.39 & 0.075 & 0.047 & 0.005 \\
\hline 16 & 272 & 74 & 0.38 & 1.15 & 0.066 & 0.064 & 0.002 & 1.40 & 0.073 & 0.070 & 0.001 \\
\hline
\end{tabular}

* These curves were corrected for catheter delay and distortion. 
material in cells is a sequestration rate. In order to provide a basis for the compilation of the data as a whole, we will assume that, in each experiment, it is appropriate to relate the calculated coefficients to the input portal vein galactose concentration. We may then explore the variation in magnitude of each parameter with substrate concentration. The limitations of these relations will be examined later.

The foregoing analysis indicates that, in exploring these phenomena, we need the plasma galactose concentration. Our measurements, however, were carried out on whole blood. Since a period of up to $24 \mathrm{~h}$ is required for the equilibration in red cell water of monosaccharides added to dog blood (10), we assume here that the galactose infused prior to each experimental run was still largely confined to the plasma space.

Assume that the product of the transport parameter and the corresponding concentration represents an initial velocity of transport $\left(\mathrm{mg} \cdot \mathrm{s}^{-1} \cdot \mathrm{ml}^{-1}\right)$; and that this process is characterized by both an asymptotic maximal velocity, $V_{g}$, and a concentration corresponding to a Michaelis-type constant, $K_{0}$. The coefficient predicted from this common relation is, in each instance, $V_{o} /$ $\left([g]+K_{0}\right)$, where $[g]$ is the substrate concentration. Optimal fitted values for $V_{0}$ and $K_{0}$ may then be obtained by minimizing the sum of the squares of the differences between the coefficients computed from the experimental data and those predicted on this basis. Initial estimates of the two parameters were obtained by use of the linearized Eadie form (11) of the hyperbolic transport relation. These were then optimized on the digital computer, by use of an iterative procedure based on Newton's method.

The variation in the two transport coefficients with plasma galactose concentration is illustrated in the upper panel of Fig. 5; and the two best fit loci are superimposed upon the illustration. For $k_{1} \theta /(1+p \gamma)$ the

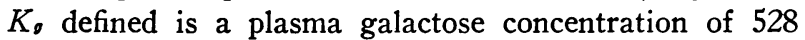
$\mathrm{mg} / 100 \mathrm{ml}$, and the $V_{g}$ is $2.20 \mathrm{mg} \cdot \mathrm{s}^{-1} \cdot \mathrm{ml}^{-1}$. For $k_{2} / r$ the $K_{g}$ is $6293 \mathrm{mg} / 100 \mathrm{ml}$; and the $V_{g}, 12.80 \mathrm{mg} \cdot \mathrm{s}^{-1}$. $\mathrm{ml}^{-1}$. The values for this second parameter scatter a great deal around the best fit line and the $K_{0}$ value lies far above the range of observed values and therefore no great confidence can be placed in the derived values of $K_{0}$ and $V_{\bullet}$, in this instance. The best fit line is almost flat, over the experimental range. The ratio of the two parameters $\left(k_{1} / k_{2}\right) r \theta /(1+p \gamma)$ is illustrated in the lower panel of Fig. 5. This ratio is higher for lower values of the plasma galactose and lower for higher values of the plasma galactose concentrations. Part of this change in the ratio with substrate concentration may be artefactual, due to changes in the liver resulting from the experimental procedure. Massive

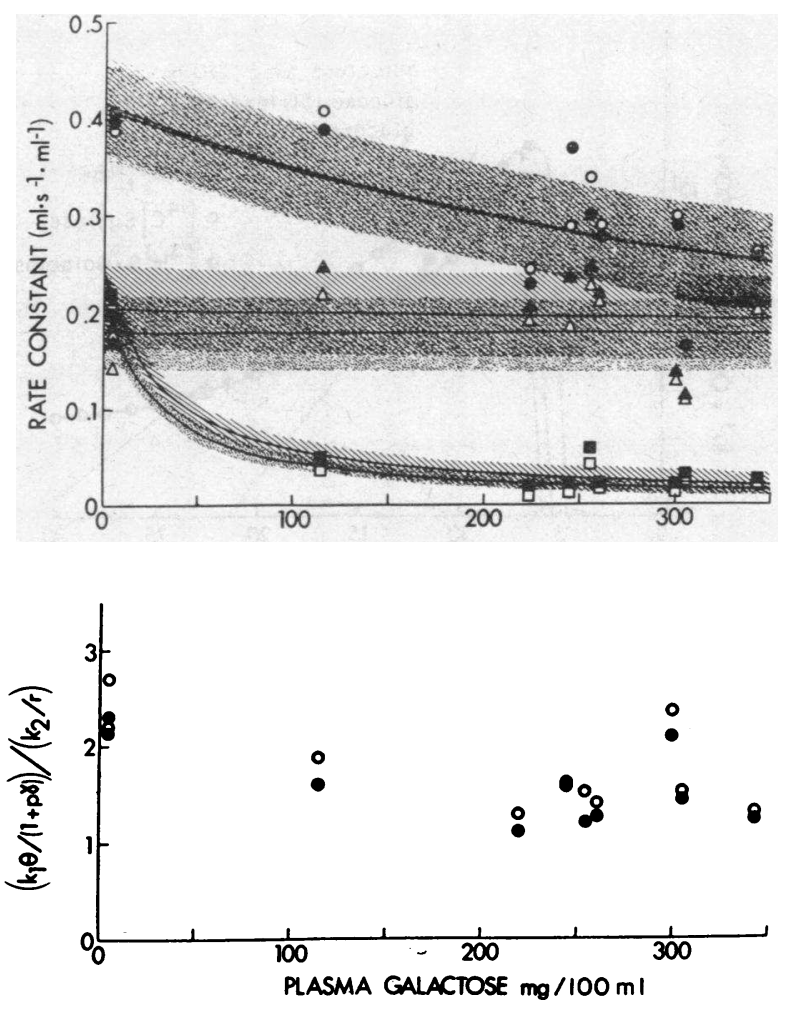

Figure 5 Upper panel. The relation between the derived parameters and the plasma galactose concentration. The parameters determined from the experimental curves were represented in the following manner: $k_{1} \theta /(1+p \gamma)$, filled circles; $k_{2} / r$, filled triangles; and $k_{3} / r$, filled squares. A range of \pm 1.0 standard error of the estimate around the best fit locus through each of these sets of points is represented by dotted shading. Values for the same parameters, determined from curves corrected for the delay and distortion introduced by the collecting system, are displayed as the corresponding open symbols. The corresponding range about the best fit loci through these sets of points is represented by hatched shading. Lower panel. The relation between the ratio $\left[\left(k_{1} \theta /(1+p \gamma) /\left(k_{2} / r\right)\right]\right.$ and the plasma galactose concentration. Values for the original curves are represented by the filled circles; and those for the corrected curves, by open circles.

galactose or glucose infusion results in an influx of fluid into the vascular space, a drop in hematocrit, and a tendency to expansion in the apparent extravascular extracellular space accessible to sucrose. However, despite this increase in the extracellular space, the pattern of decrease in the ratio with increase in the plasma galactose concentration appears real. The pattern displayed is what would have been expected if the transport mechanism were concentrative (12), a higher ratio at low substrate levels. Steady state (liver/plasma) concentration ratio values for glucose have previously been observed to approach 3.0, at low serum glucose values, and to decrease at higher values (13); and 

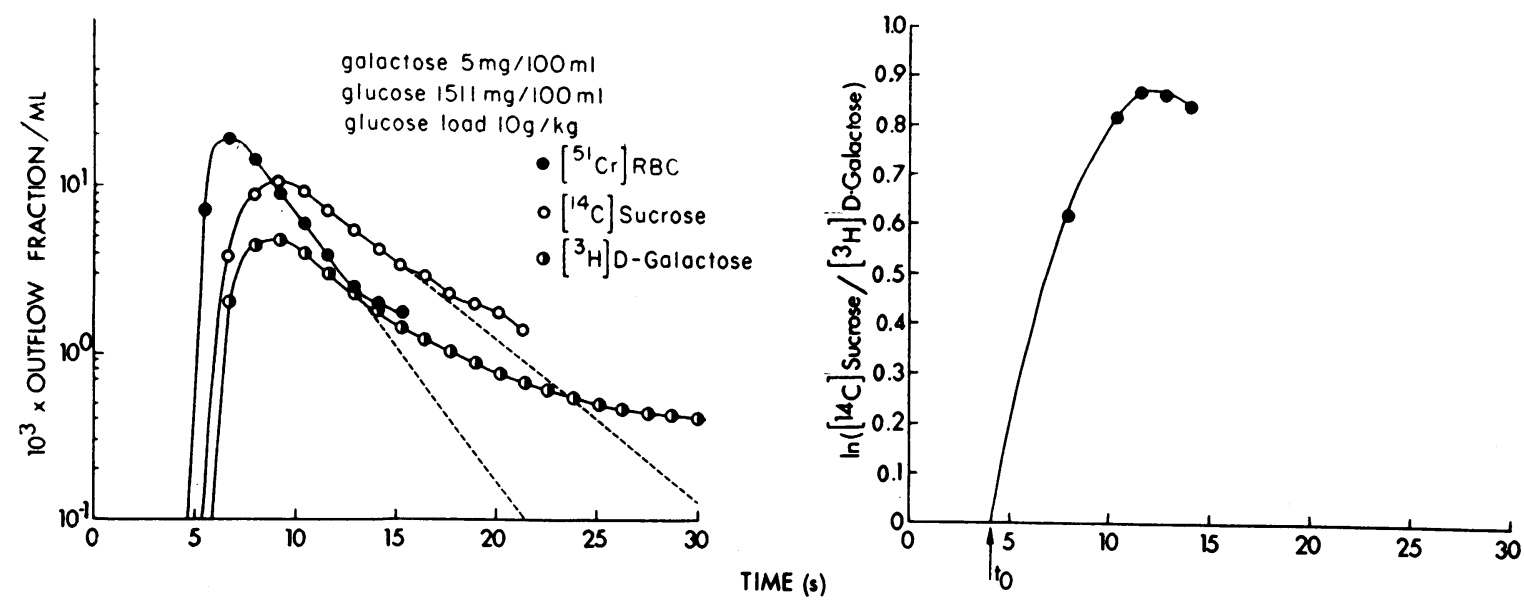

FIGURE 6 Outflow profiles for labeled red cells, sucrose, and galactose, when the blood glucose level has been grossly elevated by massive glucose loading.

so this apparent manifestation of concentrative transport of a monosaccharide may not be unexpected. At higher galactose values the process will be expected to become less concentrative and finally equilibrative. The ratio of the parameters will then theoretically begin to approach $\theta r /(1+p \gamma)$, the ratio of the cellular to the total extracellular space of distribution. This ratio value, illustrated in the lower panel of the figure, appears lower than expected, at the higher galactose concentrations.

Computed values for $k_{3} / r$ are also displayed, as a function of plasma galactose concentrations, in the upper panel of Fig. 5. In the absence of galactose loading, high values for this parameter are obtained. After loading, the value for the parameter $k_{s} / r$ becomes relatively small. The process of sequestration, the phosphorylation, would be expected to obey classical enzyme kinetics. For this parameter the fitting procedure therefore provides an estimate of the input plasma galactose concentration corresponding to the intracellular galactokinase Michaelis constant or $K_{m}$. This was found to be $32 \mathrm{mg} / 100 \mathrm{ml}$; and the maximal derived phosphorylation rate was $0.078 \mathrm{mg} \cdot \mathrm{s}^{-1} \cdot(\mathrm{ml}$ accessible intracellular space $)^{-1}$.

The effect of glucose. Massive glucose loading produces a characteristic change in the labeled galactose outflow pattern (Fig. 6). The relation between the peak of the labeled galactose curve and that of the labeled sucrose in this figure compares to that displayed at the highest galactose level (the right-hand panel of Fig. 1). Thereafter the patterns diverge. After glucose loading the labeled galactose curve decays much more quickly than it does after the galactose loading. The plot of the logarithm of the ratio (sucrose: galactose outflow fractions per milliliter) versus time exaggerates the difference in the outflow patterns. The implication of the pattern is that glucose loading decreases the transport rates of galactose across the liver cell membrane; and that, despite this, the rate of phosphorylation of galactose is fairly well preserved.

The inhibitory effect of glucose upon the transport of galactose would be expected to be competitive. The $K_{\theta}$ values computed from the transport coefficients may therefore be set equal to $K_{m}\left(1+[i] / K_{\imath}\right)$, and where $K_{m}$ is the true Michaelis-type constant for the transport of labeled galactose in the absence of glucose, and $K_{\text {, }}$ is the Michaelis-type constant characterizing the effect of the inhibitor, glucose, upon the transport process for galactose. Data from the two experiments in which no galactose was infused ( 1 and 2 ) and the two in which glucose loading ( 3 and $10 \mathrm{~g} / \mathrm{kg}$ ) was employed over the 40 min preceding the run were then analyzed. In order to simplify this analysis the galactose levels for these experiments, in the absence of loading, were assumed to approximate zero. The predicted expression for each transport coefficient then becomes $V_{g}\left(K_{i} / K_{m}\right) /$ $\left(K_{i}+[i]\right)$. The least squares method was then used to derive values for $V_{\mathfrak{g}}\left(K_{\mathfrak{t}} / K_{m}\right)$ and $K_{\mathfrak{t}}$; and, from these, values for the $K_{m}$, for each of the transport coefficients. For $k_{1} \theta /(1+p \gamma)$, the value determined for $K_{6}$ was a plasma glucose concentration of $899 \mathrm{mg} / 100 \mathrm{ml}$; and the corresponding $K_{m}$ was a plasma galactose concentration of $469 \mathrm{mg} / 100 \mathrm{ml}$. The effects of glucose upon the galactose tracer influx are small, and are grossly evident only at exceedingly high levels of blood glucose. For $k_{2} / r$, the corresponding $K_{\imath}$ and $K_{m}$ values were 675 and $6,293 \mathrm{mg} / 100 \mathrm{ml}$.

The effect of the glucose loading on $k_{3} / r$ is tabulated in Table II. The values are decreased from those expected for low serum galactose levels. However, the decrease in $k_{3} / r$ with galactose loading is relatively less than that in the transport coefficients, when the values 
TABLE III

Parameters Proitiding the Best Fit to the Coefficients Derized from Both the Original and the Corrected Curies

\begin{tabular}{|c|c|c|c|c|c|c|c|c|}
\hline \multirow[b]{2}{*}{ Coefficient } & \multicolumn{2}{|c|}{$\underset{\text { galactose }}{V_{g}}$} & \multicolumn{2}{|c|}{$\begin{array}{c}K_{\theta} \\
\text { galactose }\end{array}$} & \multicolumn{2}{|c|}{$\begin{array}{c}K_{m} \\
\text { galactose }\end{array}$} & \multicolumn{2}{|c|}{$\begin{array}{c}K_{i} \\
\text { glucose }\end{array}$} \\
\hline & Original & Corrected & Original & Corrected & Original & Corrected & Original & Corrected \\
\hline & \multicolumn{2}{|c|}{$m g s^{-1} \cdot m l^{-1}$} & \multicolumn{2}{|c|}{$m g / 100 \mathrm{ml}$} & \multicolumn{2}{|c|}{$m \mathrm{~g} / 100 \mathrm{ml}$} & \multicolumn{2}{|c|}{$m g / 100 \mathrm{ml}$} \\
\hline$k_{i} \theta^{\prime}(1+p \gamma)$ & 2.20 & 2.28 & 528 & 553 & 469 & 514 & 899 & 1,491 \\
\hline$k_{2} / r$ & 12.80 & 61.66 & 6,293 & 34,000 & 5,395 & 31,400 & 675 & 1,344 \\
\hline$k_{3} / r$ & 0.078 & 0.055 & - & - & 32 & 23 & - & - \\
\hline
\end{tabular}

are related to those of the high galactose experiments. No attempt was made to analyze this diminution, in terms of competitive inhibition, for the following reasons. Two substrates are sequentially involved in the galactose phosphorvlation process: first, a magnesium ion-A 'T' complex; and second, the galactose (14). The effect of glucose loading upon ATP generation and the first step has not been well defined; and the eifect of glucose loading upon the binding of galactose to the enzyme has not yet been reported. In our previous analysis we have implicitly assumed that the concentration of the magnesium ion-ATP complex remained constant, under the stress of galactose loading.

The effect of the collecting system. The collecting system produces delay in and distortion of the observed outflow patterns. This change in the curves would be expected to affect the magnitude of the calculated parameters. The curves were therefore corrected for the delay and distortion, by use of the inverse transfer function of the collecting system (15), and the model parameters were redetermined from these corrected curves. The average relative coefficient of variation for the fit to the corrected galactose curves was 0.048 . The changes in the model parameter estimates are illustrated in Fig. 5: and the new values for the transport parameters are listed in Table III. For $k_{1} \theta$ / $(1+p \gamma)$ the values for $V_{g}$ and $K_{g}$ increase slightly. For $k: / r$. both increase greatly, but again the curve is relatively flat over the experimental range, and the calculated values are so far above this range that one must conclude that there is little evidence for saturation of the efflux process. The $K_{i}$ value for glucose is increased; and the $K_{m}$ values for the transport process, derived by use of this higher figure, are found to be only slightly lower than the $K_{g}$ values. For the parameter $k_{i:} / r$ both the $K_{m}$ and the estimated maximal phosphorylation rate show large decreases. The $K_{m}$ decreases to a concentration corresponding to an input plasma salactose concentration of $23 \mathrm{mg} / 100 \mathrm{ml}$.

The rationale for calculating parameters from both observed and corrected data is as follows. First, these calculations make evident the magnitude of the effect of the catheter distortion upon the numerical parameters derived by application of the modeling. And secondly, the direction and perhaps the order of magnitude of the error remaining in the values estimated from the corrected data as a result of the distorting effect of the large vessels in the liver, also becomes estimable. For $k_{3} / r$ the value for $V_{g}$, derived from the corrected data is $0.048 \mathrm{mg} \cdot \mathrm{s}^{-1} \cdot \mathrm{ml}^{-1}$. The galactose elimination capacity in man has previously been shown to be approximately $0.005 \mathrm{mg} \cdot \mathrm{s}^{-1}$ (g liver) $)^{-1}(2)$ : and, since the intracellular water is approximately $0.55 \mathrm{ml} / \mathrm{g}$ (16), this value corresponds to $0.01 \mathrm{mg} \cdot \mathrm{s}^{-1} \cdot(\mathrm{ml} \text { intracellular water })^{-1}$. Our estimated value for the maximal phosphorylation capacity therefore still appears too large but the direction and a large part of the magnitude of the disparity may reasonably be explained by the large vessel distortion, in view of the major change in the values as the result of catheter distortion. Similar conclusions may be reached with regard to the apparent $K_{m}$ for galactokinase. The $K_{m}$ for the enzyme in pig liver is $10 \mathrm{mg} /$ $100 \mathrm{ml}(17)$ and it appears likely that the value for $\operatorname{dog}$ liver is similar. Correction for catheter distortion has resulted in a closer approach to the expected value. A major residual difference remains, however, and some of it is likely due to the disparity between the input plasma and the true intracellular galactose concentration at the $K_{m}$. This will be discussed later.

The derived parameters and the space approximations outlined above may be used to estimate flux ratios at the input end of the sinusoids. At a galactose level of $5 \mathrm{mg} / 100 \mathrm{ml}$ and at ordinary glucose concentrations the ratio of influx to phosphorylation is approximately 1.06 ; at galactose levels of 100,200 , and $300 \mathrm{mg} / 100$ $\mathrm{ml}$, the approximate ratios increase to 4.1 . 7.2. and 10.5 .

Phlorizin infusion. The effect of a steady intraportal infusion $(0.24 \mathrm{~g} / \mathrm{kg}$ over $30 \mathrm{~min})$ on the outflow pattern of labeled galactose is illustrated in Fig. 7. The peak of the labeled galactose curve is close to that for labeled sucrose in magnitude (i.e., the initial uptake is grossly. inhibited), and the labeled galactose curve steadily di-

Uptake of Galactose By the Intact Liver 


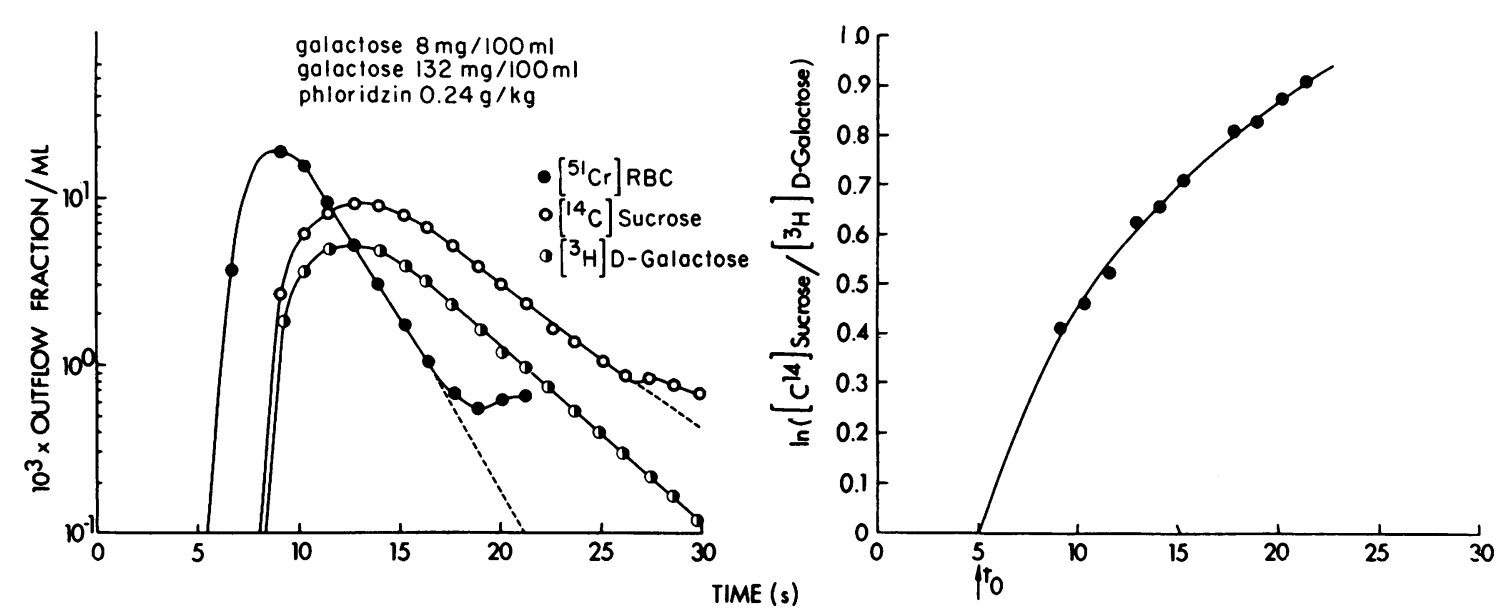

FIGURE 7 Outflow patterns for labeled red cells, sucrose, and galactose, when phlorhizin is being infused intraportally.

verges from that of labeled sucrose. The natural logarithm of the ratio of the labeled sucrose to galactose outflow fractions per milliliter increases steadily. The parameters $k_{1} \theta /(1+p \gamma)$ and $k_{2} / r$ are low, in relation to the values previously obtained at low galactose concentrations. The value for $k_{3} / r$ is also decreased but not proportionately. It remains high enough that essentially all of the labeled galactose entering each cell is phosphorylated. The throughput component of the outflow pattern is therefore emphasized, just as it is in a highly concentrative transport system, in which material which has entered the cells returns to the flowing blood very slowly (5).

$\mathrm{L}$-galactose, $\beta$-methyl $\mathrm{D}$-galactoside, and methyl-1thio- $\beta$-D-galactoside. The experiments in this section exemplify the manner in which changes in molecular structure alter the affinity of the molecular for the

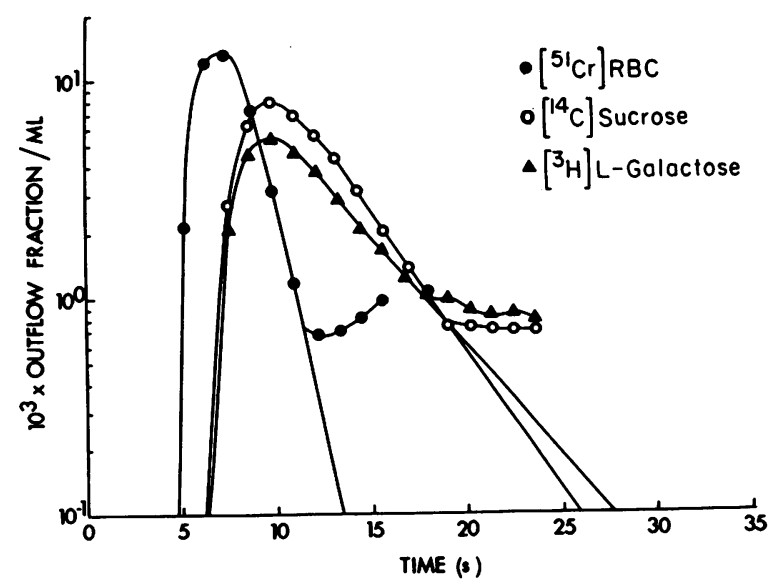

Figure 8 Outflow patterns for labeled red cells, sucrose, and L-galactose. A load of D-galactose has been infused in this instance. transport system subserving entry into and exit from the liver cells. The behaviour of L-galactose, the mirror image of D-galactose, was explored. In Fig. 8, the outflow patterns from an L-galactose experiment are displayed. The peak of the labeled L-galactose curve is once again close to that of the labeled sucrose curve in magnitude, but on the downslope the L-galactose curve approaches and crosses the labeled sucrose curve. The material entering the cells returns rapidly and virtually completely to the perfusing blood and to the outflow. The calculated proportion irreversibly sequestrated is almost negligible. The level of $\mathrm{D}$-galactose appears to have no effect on the transport of the labeled L-galactose (see Table II). The implications of these studies are that the L-isomer enters the liver cells much more slowly than the D-isomer, and that metabolic utilization of this compound is either negligible or very slow.

We have previously shown that $\beta$-methyl $D$-glucoside is virtually identical to labeled sucrose, in its space of distribution (18). The outflow pattern for $\beta$-methyl $\mathrm{D}$ galactoside superimposes on that of $\beta$-methyl D-glucoside. The replacement of the equatorial $\beta$-hydroxyl group of the $\mathrm{Cl}$ form of the galactopyranoside has resulted in loss of the affinity of the molecule for the galactose transport system. Similarly, the methyl-1-thio- $\boldsymbol{\beta}$-D-galactoside is also not transported at a significant rate by the galactose carrier system.

\section{DISCUSSION}

Utility of this approach. This study creates for the first time a methodology directed at separating and quantifying the relative roles of a transport process and an enzymatic intracellular sequestration process, in the disposal of a substance by the liver. The procedures developed will potentially be of use in clinical investigation and provide a basis for the nondestructive in vivo esti- 


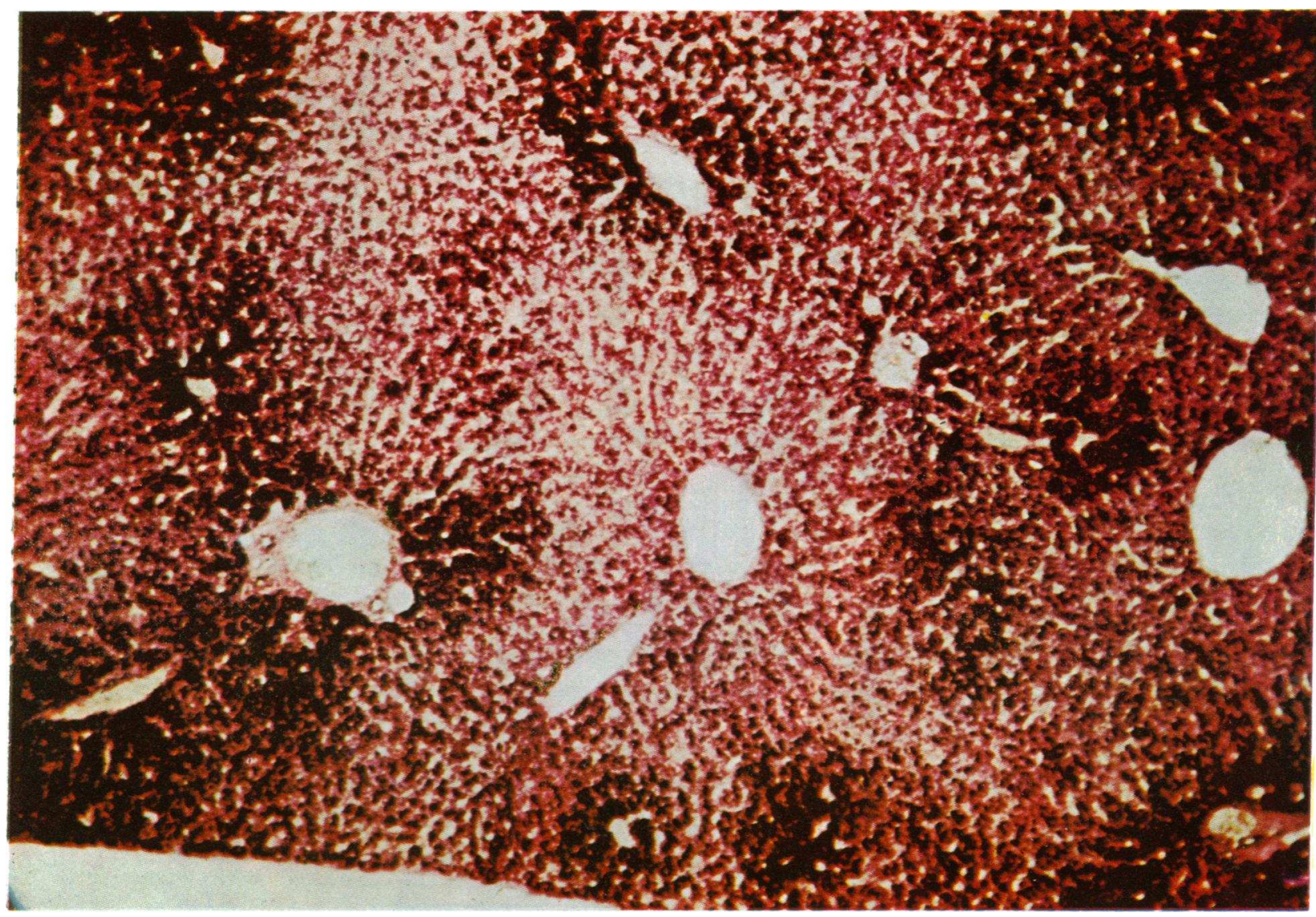

Figure 9 The lobular intracellular concentration gradient. Tritium-labeled galactose was injected intravenously in trace dose into a suckling mouse and this liver sample was removed 2 min later. During this short interval a portion of labeled galactose entered the cell, was phosphorylated and isomerized to form galactose-1-phosphate, and was then incorporated into glycogen. During fixation of the tissue the water soluble hexose and hexose-phosphates were washed away and only the glycogen polymer remained behind. The slide was stained with periodic acid-Schiff, which delineates glycogen as pink clumps and an autoradiograph was then developed in an overlying layer of emulsion. There is clearly a large concentration of radioactivity in the area surrounding the portal triads and this decreases in intensity in the direction of the central vein. We wish to express our appreciation to Dr. Gary Bennett of the McGill Department of Anatomy, who carried out these preparations.

mation of the parameters characterizing the two processes.

Adequacy of the analysis used here. The single element used in the model analysis is essentially an isolated Krogh type of cylinder. We have explored elsewhere (19) the phenomena underlying the distribution of labeled water in the liver and have concluded that there is no experimental evidence to indicate that major diffusional transport of this material occurs along the length either directly within the element or by diffusional interconnection by virtue of staggered entrance and exit architecture. For galactose, where such effects would be smaller, we may therefore conclude that the relatively good fit of the predicted to the experimental curves, and the corre- spondence of the estimates of the maximal rate of phosphorylation and of the $K_{m}$ value for this process to the kinds of values expected, indicate that a fair degree of confidence may be placed in the modeling outlined in this paper. The modeling appears to provide an appropriate description of the data despite the other simplifications inherent in our formulations (the assumption of uniform large vessel transit times, and of large scale homogeneity of perfusion in the liver).

Lobular concentration gradients. One of the major inferences of the modeling developed in the Appendix is the presence of a lengthwise concentration gradient, from portal triad to central vein, in both the sinusoids and the cells when a process of irreversible intracellular seques-

Uptake of Galactose By the Intact Liver 
tration takes place. Two important aspects of the liver morphology contribute to this phenomenon: the liver is quasi-crystalline in structure, with input and output points symmetrically arranged: and all of the entrances and exists from sinusoids are adjacent, so that there is no opportunity in the structure for diffusional shortcircuiting. The presence of the lobular concentration gradients are so integral a part of the modeling utilized here that, if they were not present, grave doubt could be cast upon the adequacy of the assumptions utilized. We therefore examined radioautographic exposures on sections of liver taken shortly after the administration of labeled galactose. One is displayed in Fig. 9. The expected lobular concentration gradient is unequivocally present after the administration of labeled galactose.

The lobular concentration gradient creates another kind of difficulty, an uncertainty in the definition of the $K_{\text {n }}$ for the intracellular sequestration process. In our analysis we have, of necessity, related the net changes in intracellular sequestration across the whole liver to the input concentration of galactose and have derived an apparent $K_{m}$ value. The value which we wish is, however, the average lobular intracellular concentration at the apparent $K_{n}$. We can extrapolate to this in the following manner. The plasma value at the apparent $K_{m}$ was 23 $\mathrm{mg} / 100 \mathrm{ml}$, if we neglect the residual remaining large vessel distortion. At this input concentration the average extraction across the lobule approximates $90 \%$ and so the average corresponding sinusoidal concentration will be approximately $15 \mathrm{mg} / 100 \mathrm{ml}$. At the same time, there will be a stepdown in concentration across the cell membrane at each point along the sinusoid and so the average intracellular concentration will be even less. It will be of the order of $10 \mathrm{mg} / 100 \mathrm{ml}$. Similarly, it is obvious that the tissue gradient will impose the same kind of difficulties if biopsy material is used to determine the $K_{m}$.

The effect of glucose. The effect of glucose on the transfer of galactose into liver cells is not unexpected. The converse phenomenon has previously been docimented in the brain. D-glucose leaves the rather impermeable cerebral capillaries by means of a stereospecific carrier transport mechanism (20); and galactose loading produces a major and significant inhibition of the penetration of tracer glucose into the brain (21).

Concentrative uptake of material by the liver, with intracellular removal. Concentrative uptake may occur either because there is a high concentration of binding substance inside the cells, with a high affinity for the transported material (22), or because of the intrinsic rate constant for return is less than that for entrance (i.e., because the transport process itself is concentrative). These two possibilities cannot be distinguished by means of a kinetic examination of the uptake process. Either results in a second component, a tail function, which is reduced in magnitude and prolonged in time; and, concomitantly, in a relative accentuation of the throughput component. If, at the same time, these conditions coexist with a situation in which the rate constant for sequestration is relatively large, the outflow profile may appear to consist almost exclusively of throughput material. Experimentally, the handling of trace doses of sulfobromophthalein has been found to correspond to this case (23). If, however, a steady infusion of sulfobromophthalein had been administered prior to the tracer study, so that the sequestering process (biliary secretion) became saturated, the return of material from cells to plasma would have been expected to become evident. If, in this situation, the amount of binding substance was experimentally increased (24), this return to plasma would be expected to be retarded; and the apparent removal rate, to be increased, when it is calculated by use of data collected at times greater than that of a single passage.

The use of the present modeling to describe cxchange across a capillary, with apparently irreversible uptake of the exchanging matcrial by parenchymal cells. If, in the present modeling, we set the extracellular extravascular space equal to zero, and the rate constants for uptake and exit per unit area to a permeability, it may be used to describe exchange across a capillary permeable to the substance under consideration, when the exchanging substance is removed from the extracellular space in a manner which is essentially irreversible over the time being considered. This will occur when the substance being considered is either consumed or removed from the extracellular space in a concentrative fashion by the surrounding parenchymal cells. Thus, for instance, the equation will describe the concentrative uptake of labeled $\mathrm{Rb}^{+}$or $\mathrm{K}^{+}$by muscle cells, over early times after injection, when essentially none of the label is returning to the extracellular space (see, for example, reference 25).

Change in the estimated transport parameters with flow. We previously analyzed the concentrative uptake of labeled $\mathrm{Rb}^{+}$by the hepatic parenchymal cells (5). The early labeled $\mathrm{Rb}^{+}$outflow curves consisted almost completely of throughput material. Fairly reliable values of the parameter $k_{1} \theta /(1+p \gamma)$ were obtained. Over an approximately fourfold range of flows $(0.014-0.062 \mathrm{ml}$. $\left.\mathrm{s}^{-1} \cdot \mathrm{g}^{-1}\right)$, no major change in this transport parameter occurred. The liver appears to be fairly uniformly perfused over this range, and no major recruitment of new sinusoids (i.e., of liver cell transport surface) seems to occur, with increase in flow. In contrast, the analysis of dilution patterns from the working heart, obtained at varying perfusion rates, has yielded a completely different pattern. As the perfusion is increased, there 
is a recruitment of capillary surface, and an increase in the permeability surface product subserving exchange (26). Despite this pattern of response, the spatial distribution of capillary perfusion is such that materials from the working capillaries appear to reach all fibers. The parameter describing uptake of labeled $\mathrm{Rb}^{+}$at the muscle cell surface does not change with flow, and so the proportion of the muscle cell surface utilized for transport does not appear to vary with flow. The lack of change in transport surface with flow, in the liver, appears to have its parallel in the lack of change in effective muscle cell transport surface with flow, in the heart.

Potential future applications of the present analysis. The modeling applied in this paper to analyze the outflow pattern for galactose can be used to study the simultaneous processes of cellular transport and either metabolic sequestration or biliary excretion. Its application to the analysis of the behavior of compounds undergoing metabolic sequestration is straight forward and has been outlined above. Its application to studies of substances undergoing biliary excretion will be equally simple, if the properties of the compound being excreted are not changed by the process of intracellular conjugation. If a process of intracellular conjugation is part of the metabolic handling of the compound, the kinetics will be somewhat more complex if the intracellular binding of the conjugated compound is different, and if the affinity of the compound for the transport systems subserving cellular entry and exit is different than that of the parent compound. If the changes in these parameters produced by conjugation are minor, they can be neglected, and the present modeling will be applicable.

\section{APPENDIX}

The equations for a single sinusoid. Once again (5), consider a sinusoid of length $L$ in which blood flows with a velocity $W$, and which is enfolded by an extracellular space, the space of Disse, with which free communication occurs, so that soluble substances in the sinusoidal plasma will undergo flowlimited distribution into this space. In this model let $W=$ the velocity of flow in the sinusoid; $u(x, t)=$ the concentration in the sinusoid at some point along the length $x$, at the time $t$; $v(x, t)=$ the corresponding concentration in the adjacent extracellular space; $z(x, t)=$ the concentration of unsequestered material in the cells, which is potentially able to reappear in the blood; $A, B, C=$ the volume per unit length for sinusoidal, extracellular space, and cellular space, respectively (they are regarded as constant along the length); $B / A=\gamma$, and $C / A=\theta ; p, r=$ the partition coefficients for the substance under consideration in the extracellular space and intracellular space, with respect to the corresponding equilibrium plasma concentration (these parameters describe the relative solubilities of the substance in those phases); and $k_{3}=$ the rate constant for the sequestration process, with the dimensions time ${ }^{-1}$.

The conservation equation (developed by equating the change in quantity of material in the system between any $x$ and $x+\Delta x$ during a fixed time interval $\Delta t$ to the quantity of material sequestered in the cells and the change in the quantity of material in the sinusoid) is

$$
\frac{\partial u}{\partial t}+W \frac{\partial u}{\partial x}+\gamma \frac{\partial v}{\partial t}+\theta \frac{\partial z}{\partial t}+\frac{k_{3}}{r} \theta z=0
$$

With the assumption of flow-limited distribution in the extracellular space $v(x, t)=p u(x, t)$ and $\partial v / \partial t=p \partial u / \partial t$, and hence

$$
\frac{(1+p \gamma)}{W} \frac{\partial u}{\partial t}+\frac{\partial u}{\partial x}+\frac{\theta}{W} \frac{\partial z}{\partial t}+\frac{k_{3} \theta z}{r W}=0
$$

We may now formulate an equation describing both the transport of material between the extracellular space and cellular interior, and the sequestration process. Since the transport process may be asymmetric, two transport rate constants will be used. The rate of cellular influx of material is assumed proportional to the product of the cell surface per unit length $\phi$, the length under consideration $\Delta x$, the rate constant for uptake per unit area $k_{1}{ }^{\prime}$ (with dimensions $\mathrm{cm} \cdot \mathrm{s}^{-1}$ ), and the product of $p^{-1}$ and the extracellular concentration $v(x, t)$; the rate of efflux of material to the extracellular space, proportional to the product $\phi \Delta x$, the rate constant for exit per unit area $k_{2}{ }^{\prime}$ (with dimensions $\mathrm{cm} \cdot \mathrm{s}^{-1}$ ), and the product of $r^{-1}$ and the cellular concentration $z(x, t)$; and the rate of sequestration, proportional to the product of the uptake rate constant $k_{3}$, the element of cellular volume $C \Delta x$, and the product of $r^{-1}$ and the cellular concentration $z(x, t)$. Whence

$$
\begin{aligned}
\frac{\partial z}{\partial t} & =\frac{k_{1}{ }^{\prime} \phi}{C} \frac{v}{p}-\frac{k_{2}{ }^{\prime} \phi}{C} \frac{z}{r}-k_{3} \frac{z}{r} \\
& =k_{1} u-k_{2} \frac{z}{r}-k_{3} \frac{z}{r}
\end{aligned}
$$

where $k_{1}=k_{1}{ }^{\prime} \phi / C$, and $k_{2}=k_{2}{ }^{\prime} \phi / C$. We will again consider the transport system to be an equilibrative one when $k_{1}{ }^{\prime}=k_{2}{ }^{\prime}$, a concentrative one when $k_{1}{ }^{\prime}>k_{2}{ }^{\prime}$.

Solution of the equations for a single sinusoid. Once again introduce at the origin $(x=0)$ of the initially empty sinusoid with the flow $F_{s}$, the quantity of material $q_{0}$, at the time $t=\epsilon$ (a value infinitesimally greater than zero). Equations 1 and 2 must then be solved according to the initial conditions

$$
\begin{aligned}
& u(0, t)=\frac{q_{0}}{F_{s}} \delta(t-\epsilon) \\
& u(x, 0)=0, z(x, 0)=0, \text { and } u^{\prime}(x, 0)=0,
\end{aligned}
$$

where $\delta(t-\epsilon)$ is a Dirac delta function or impulse function. Eliminating $z$ from equations 1 and 2 we find

$$
\begin{array}{r}
\frac{\partial^{2} u}{\partial t^{2}}+\left(\frac{W}{1+p \gamma}\right) \frac{\partial^{2} u}{\partial x \partial t}+\left[\frac{k_{1} \theta}{1+p \gamma}+\frac{k_{2}}{r}+\frac{k_{3}}{r}\right] \frac{\partial u}{\partial t} \\
+\left[\frac{k_{2}}{r}+\frac{k_{3}}{r}\right] \frac{W}{(1+p \gamma)} \frac{\partial u}{\partial x}+\left(\frac{k_{1} \theta}{1+p \gamma}\right) \frac{k_{3}}{r} u=0
\end{array}
$$

Appropriately, when $k_{3}=0$ this equation becomes that de-

Uptake of Galactose By the Intact Liver 
scribing the phenomena of transport, which we derived earlier (5).

Now substitute $u(x, t)=e^{\beta x} m(x, t)$. Then we find that, for the value $\beta=-k_{1} \theta k_{3} / I V\left(k_{2}+k_{3}\right)$, the equation becomes

$$
\begin{gathered}
\frac{\partial^{2} m}{\partial t^{2}}+\frac{W}{(1+p \gamma)} \frac{\partial^{2} m}{\partial x \partial t} \\
+\left[\frac{k_{1} \theta}{1+p \gamma}+\frac{k_{2}}{r}+\frac{k_{3}}{r}-\frac{k_{1} \theta}{(1+p \gamma)} \cdot \frac{k_{3}}{\left(k_{2}+k_{3}\right)}\right] \frac{\partial m}{\partial t} \\
+\left(\frac{k_{2}}{r}+\frac{k_{3}}{r}\right) \frac{W}{(1+p \gamma)} \frac{\partial m}{\partial x}=0
\end{gathered}
$$

Applying the Laplace transformation

$$
\mathcal{L}(m[x, t])=\int_{0}^{\infty} m(x, t) \exp (-s t) d t=\bar{M}(x, s)
$$

we find

$$
\begin{aligned}
\bar{M}(x, s)= & \bar{M}(0, s) \exp \left[-s(1+p \gamma) \frac{x}{W}\right] \\
& \times \exp \left(-k_{1} \theta \frac{x}{W}\right) \exp \left[\frac{k_{1} \theta k_{3}}{W\left(k_{2}+k_{3}\right)}\right] \cdot x \\
& \times \exp \left\{\frac{x}{W}\left[\left(k_{1} \frac{k_{2}}{r}\right) /\left(s+\frac{k_{2}}{r}+\frac{k_{3}}{r}\right)\right]\right\}
\end{aligned}
$$

From which

$$
\begin{aligned}
\bar{U}(x, s)= & \bar{U}(0, s) \exp \left[-s(1+p \gamma) \frac{x}{W}\right] \exp \left[-k_{1} \theta \frac{x}{W}\right] \\
& \times \exp \left\{\frac{x}{W}\left[\left(k_{1} \theta \frac{k_{2}}{r}\right) /\left(s+\frac{k_{2}}{r}+\frac{k_{3}}{r}\right)\right]\right\} \\
= & \bar{U}(0, s) \exp \left[-s(1+p \gamma) \frac{x}{W}\right] \\
& \times \exp \left(-k_{1} \theta \frac{x}{W}\right)\left(1+\frac{k_{1} \theta \frac{k_{2}}{r} \frac{x}{W}}{\left(s+\frac{k_{2}}{r}+\frac{k_{3}}{r}\right)}\right. \\
& \left.+\frac{\left(k_{1} \theta \frac{k_{2}}{r} \frac{x}{W}\right)^{2}}{2 !\left(s+\frac{k_{2}}{r}+\frac{k_{3}}{r}\right)^{2}}+\cdots\right)
\end{aligned}
$$

Whence for the impulse input

$$
\begin{gathered}
u(x, t)=\frac{q_{0}}{F_{s}} \exp \left(-k_{1} \theta \frac{x}{W}\right) \delta\left(t-[1+p \gamma] \frac{x}{W}\right) \\
+\frac{q_{0}}{F_{s}} \exp \left[-\left(\frac{k_{2}}{r}+\frac{k_{3}}{r}\right)\left(t-[1+p \gamma] \frac{x}{W}\right)\right] \\
\times \exp \left(-k_{1} \theta \frac{x}{W}\right) \sum_{n=1}^{\infty} \frac{\left(k_{1} \theta \frac{k_{2}}{r} \frac{x}{W}\right)^{n}\left(\left(t-[1+p \gamma] \frac{x}{W}\right)^{n-1}\right.}{n !(n-1) !} \\
\times S\left(\left(t-[1+p \gamma] \frac{x}{W}\right)\right.
\end{gathered}
$$

where $S\left(t-[1+p \gamma] \frac{x}{W}\right)$ is a step function at $t=(1+p \gamma) \frac{x}{W}$. If we wish to examine the profile at the outflow and we define the vascular reference transit time $L / W=\tau$, the expression becomes

$$
\begin{aligned}
u(L, t)= & \frac{q_{0}}{F_{s}} \exp \left(-\frac{k_{1} \theta t}{1+p \gamma}\right) \delta(t-[1+p \gamma] \tau) \\
& +\frac{q_{0}}{F_{s}} \exp \left[-\left(\frac{k_{2}}{r}+\frac{k_{3}}{r}\right)(t-[1+p \gamma] \tau)\right] \\
& \times \exp \left(-k_{1} \theta \tau\right) \sqrt{\frac{k_{1} \theta \frac{k_{2}}{r}}{t-(1+p \gamma) \tau}} \\
& \times \mathrm{I}_{1}\left(2 \sqrt{k_{1} \theta \frac{k_{2}}{r} \tau(t-[1+p \gamma] \tau)}\right)
\end{aligned}
$$

where $I_{1}(z)$ is a first order modified Bessel function of argument $z$. It should be noted that none of the material emerges at the outflow until the time $t=(1+p \gamma) \tau$.

The relations between the outflow profile for this substance, which is undergoing both transport and sequestration or removal, and those of the reference tracers or a substance undergoing transport but not removal will now be examined. When the substance under consideration is the vascular reference substance $\left(\gamma=0\right.$ and $\left.k_{1}=k_{2}=k_{3}=0\right)$, the outflow profile is an impulse emerging at the time $\tau$. When it is a substance undergoing flow-limited distribution into the extracellular space ( $\gamma$ finite and $\left.k_{1}=k_{2}=k_{3}=0\right)$, the profile consists of an impulse function emerging at the time $(1+p \gamma) \tau$. When the substance is undergoing only transport but no removal $\left(\gamma, k_{1}\right.$, and $k_{2}$ finite; $k_{3}=0$ ) the profile consists of two parts: a first term or "spike", an impulse function emerging at the time $(1+p \gamma) \tau$, which is damped by the term $\exp \left(-k_{1} \theta \tau\right)$; and a second term or "tail function". The first term represents through put material, material which has swept by the cell surface but has not entered the cells (the amount of material in this damped spike is $\left.q_{0} \exp \left(-k_{1} \theta t /[1+p \gamma]\right)\right)$; and the second term, material which has entered and left the cells. In the present case $\left(\gamma, k_{1}, k_{2}\right.$, and $k_{3}$ finite), the expression describing the first term or throughput material remains the same. The intracellular events do not affect the initial uptake process. The tail function, on the other hand, is now damped by an addi- 


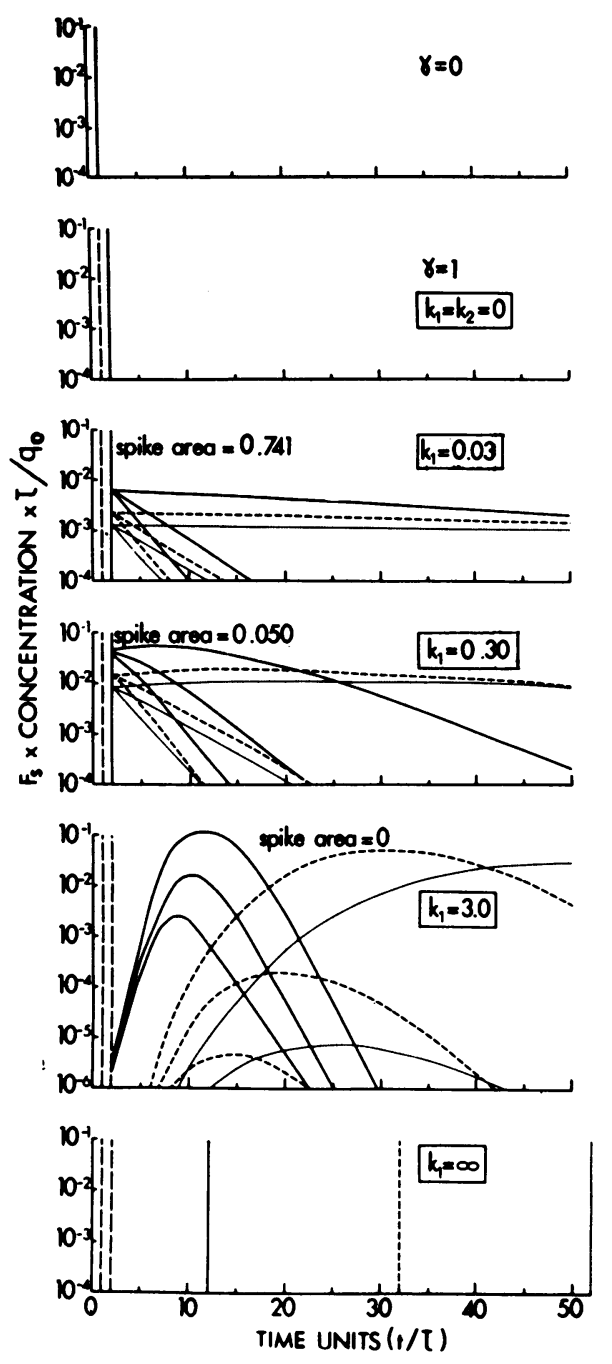

Figure 10 Computed outflow profiles. The profile in the upper panel corresponds to the vascular reference, labeled red cells; that in the second, to the extracellular reference; and those in the lower panels, to substances undergoing intracellular transport from the extracellular space. In each instance the input has been an impulse function $q_{0}$. The abscissa has been normalized to $t / \tau$ and the ordinate, correspondingly, to $F_{s} u(L, t) / q_{0}$ so that on square grid paper the areas under the outflow profile for the case in which $k_{3}=$ 0.0 would be unity. The ordinate scales in the illustration are semilogarithmic. In this illustration we have used a vertical line to represent an impulse function; and in the third, fourth, and fifth panels, have indicated the associated area by the term spike area, since it differs from unity. For the exchanging substance separate tail functions have been plotted for the $k_{1} / k_{2}$ values $1.0,3.0$, and 5.0 (the corresponding loci are solid, dashed, and dotted). In addition, we have illustrated, for each of these $k_{1} / k_{2}$ values, three profiles corresponding, from above downwards, to the $k_{3}$ values, zero, 0.25 , and 0.50 time units $^{-1}$. In the bottom panel are illustrated the three delayed wave flow-limited cases: impulse functions emerging at 12, 32, and 52 normalized time units. The area associated with each of these impulse tional term, $\exp \left(-k_{3}[t-(1+p \gamma) \tau]\right)$. This is the result of the cellular process which, with the passage of time, sequestrates an increasing proportion of the material which has entered the cells.

It is also appropriate to express equation $6 \mathrm{~A}$ in alternate form. Using the equivalent expressions for the rate constants $k_{1}$ and $k_{2}$ and defining $\phi L=S_{s}$ (the surface area of the liver cells subserving transport), and $C L=V_{c l}$ (the volume of the liver cells corresponding to the sinusoid) we find

$$
\begin{aligned}
& u(L, t)=\frac{q_{0}}{F_{s}} \exp \left(-\frac{k_{1}{ }^{\prime} S_{s}}{F_{s}}\right) \delta(t-[1+p \gamma] \tau) \\
& +\frac{q_{0}}{F_{s}} \exp \left(-\frac{k_{1}{ }^{\prime} S_{s}}{F_{s}}\right) \\
& \times \exp \left[-\left(\frac{k_{2}{ }^{\prime} S_{s}}{r V_{c l}}+\frac{k_{3}}{r}\right)(t-[1+p \gamma] \tau)\right] \\
& \times \sum_{n=1}^{\infty} \frac{\left(\begin{array}{c}
k_{1}{ }^{\prime} S_{s} \\
\left.F_{s} \frac{k_{2}{ }^{\prime} S_{s}}{r V_{c l}} \tau\right)^{n}(t-[1+p \gamma] \tau)^{n-1}
\end{array}\right.}{n !(n-1) !} \\
& \times S(t-[1+p \gamma] \tau) \text {. }
\end{aligned}
$$

Within this expression the first term, describing throughput, is dependent only on the flow and the area of the transport surface. It is independent of the cellular volume and is analogous to the expression developed by Renkin (27) and by Crone (28) to describe the emergence of throughput material from a permeable capillary. The second term, the tail function, is progressively reduced by the sequestration process.

Now consider the flow-limited case $\left(k_{1}, k_{2}\right.$ approach infinity, while the ratio $k_{1} / k_{2}$ remains constant; $k_{3}$ finite). An equivalent form of the transform may be shown to be

$$
\begin{aligned}
\bar{U}(L, s)= & \frac{q_{0}}{F_{s}} \exp \{-s(1+p \gamma) \\
& \left.\times\left[1+\left[k_{1} \theta /(1+p \gamma)\right] /\left(s+\frac{k_{2}}{r}+\frac{k_{3}}{r}\right)\right]\right\} \\
& \quad \times \exp \left[-\left(k_{1} \theta \frac{k_{3}}{r} \tau\right) /\left(s+\frac{k_{2}}{r}+\frac{k_{3}}{r}\right)\right] .
\end{aligned}
$$

The values of $s$ and of $k_{3} / r$ in the denominators of the exponents may be neglected as $k_{2} / r$ approaches infinity. Whence, for this case, we find

$$
\begin{aligned}
u(L, t)=\frac{q_{0}}{F_{s}} \exp \left(-\frac{k_{1} k_{3} \theta \tau}{k_{2}}\right) & \\
& \times \delta\left(t-\left[1+p \gamma+\frac{k_{1} \theta r}{k_{2}}\right] \tau\right)
\end{aligned}
$$

The outflow profile has become a delayed spike, propagating both within the extracellular and cellular spaces, and damped by the intracellular removal process.

functions is unity, when $k_{3}$ is zero. When $k_{3}$ assumes the values of 0.25 and 0.50 (time units) ${ }^{-1}$, the areas associated with the first impulse function become 0.087 and 0.007 , respectively; and for the latter two impulse functions, it becomes virtually zero, in both cases. This illustration is an extension of Fig. 3 of reference 5.

Uptake of Galactose By the Intact Liver 
In order to portray the phenomena which we have described above, we have computed on a digital computer a set of illustrations for the case in which $\gamma=1.0, \theta=1.0, \tau=1.0$, the partition coefficients $p$ and $r$ equal unity, and $k_{3}$ assumes three values: zero, 0.25 , and 0.50 (normalized time units) ${ }^{-1}$. These are displayed in Fig. 10. As $k_{1}$ increases the proportion of the response in the tail function increases; and as $k_{1}$ and $k_{3}$ increase, there is a remarkable change in the shape of this profile.

Conservation of matter in the single sinusoid. When $k_{3}$ has finite values the total of the material emerging at the outflow is less than $q_{0}$ by the amount sequestered by the cellular uptake process. The total of the material emerging at the outflow is

$$
\begin{aligned}
F_{s} \int_{0}^{\infty} u(L, t) d t \\
=q_{0} \int_{0}^{\infty} \exp \left(-k_{1} \theta \tau\right) \delta(t-[1+p \gamma] \tau) d t \\
\quad+q_{0} \exp \left(-k_{1} \theta \tau\right) \exp \left[\left(\frac{k_{2}}{r}+\frac{k_{3}}{r}\right)(1+p \gamma) \tau\right] \\
\quad \times \sum_{n=1}^{\infty}\left(k_{1} \theta \frac{k_{2}}{r} \tau\right)^{n} \int_{(1+p \gamma) \tau}^{\infty} \exp \left[-\left(\frac{k_{2}}{r}+\frac{k_{3}}{r}\right) t\right] \\
\quad \times\left[(t-[1+p \gamma] \tau)^{n-1} / n !(n-1) !\right] d t \\
\quad=q_{0} \exp \left[-\left(k_{1} \theta \frac{k_{3}}{r} \tau\right) /\left(\frac{k_{2}}{r}+\frac{k_{3}}{r}\right)\right] .
\end{aligned}
$$

The amount removed during passage along the single sinusoid is therefore

$$
q_{0}\left[1-\exp \left(-k_{1} \theta \frac{k_{3}}{r} \tau\right) /\left(\frac{k_{2}}{r}+\frac{k_{3}}{r}\right)\right] .
$$

Mean transit time for the material recovered at the exit. If all the material entering the cells returns to the outflow, the product of the flow and the mean transit time yields its equivalent volume of distribution. In the case which we are considering two possible modes of exit (29) from the system occur: either the material is sequestrated and converted, or it emerges at the outflow as labeled galactose. The mean sinusoidal transit time for the latter moiety, the material collected at the outflow is

$$
i=\frac{\int_{0}^{\infty} t u(L, t) d t}{\int_{0}^{\infty} u(L, t) d t} .
$$

The denominator is seen to be

$$
\left(q_{0} / F_{s}\right) \exp \left(-k_{1} \theta \frac{k_{3}}{r} \tau /\left[\frac{k_{2}}{r}+\frac{k_{3}}{r}\right]\right) .
$$

The numerator is

$$
\begin{aligned}
\int_{0}^{\infty} & t u(L, t) d t \\
= & \frac{q_{0}}{F_{s}} \int_{0}^{\infty} t \exp \left(-\frac{k_{1} \theta t}{1+p \gamma}\right) \delta(t-[1+p \gamma] \tau) d t \\
& \quad+\frac{q_{0}}{F_{s}} \exp \left(-k_{1} \theta \tau\right) \exp \left[\left(\frac{k_{2}}{r}+\frac{k_{3}}{r}\right)(1+p \gamma) \tau\right]
\end{aligned}
$$

$$
\begin{aligned}
& \times \sum_{n=1}^{\infty} \frac{\left(\frac{k_{1} \theta k_{2} \tau}{r}\right)^{n}}{n !(n-1) !} \\
& \times \int_{(1+p \gamma) \tau}^{\infty} t \exp \left[-\left(\frac{k_{2}}{r}+\frac{k_{3}}{r}\right) t\right] \\
& \times(t-[1+p \gamma] \tau)^{n-1} d t \\
& =\frac{q_{0}}{F_{s}}\left(1+p \gamma+\frac{k_{1} \theta \frac{k_{2}}{r}}{\left(\frac{k_{2}}{r}+\frac{k_{3}}{r}\right)^{2}}\right] \tau \\
& \times \exp \left[-\left(k_{1} \theta \frac{k_{3}}{r}\right) /\left(\frac{k_{2}}{r}+\frac{k_{3}}{r}\right)\right] .
\end{aligned}
$$

Whence

$$
\dot{t}=\left[1+p \gamma+\frac{k_{1} \theta \frac{k_{2}}{r}}{\left(\frac{k_{2}}{r}+\frac{k_{3}}{r}\right)^{2}}\right) \tau .
$$

As $k_{3}$ decreases the mean transit time increases until in the limit, when $k_{3}=0$, the mean transit time is $\left[1+p \gamma+k_{1} \theta r\right]$ $\left.k_{2}\right] \tau$. In this limit the product of the flow and the transit time corresponds to the total equivalent volume of distribution accessible to galactose.

The concentration profile within the sinusoidal and extracellular spaces, and within the hepatic cells: the basis for the histological zonation observed within a hepatic lobule. The concentration profile within the sinusoidal space is described by equation 6. We must develop an expression for the adjacent profile for the intracellular tracer. From equation 2 we find

$$
\bar{Z}(x, s)=\frac{k_{1} \bar{U}(x, s)}{\left(s+\frac{k_{2}}{r}+\frac{k_{3}}{r}\right)} .
$$

Substituting,

$$
\begin{aligned}
\bar{Z}(x, s)= & \frac{k_{1} \bar{U}(0, s)}{\left(s+\frac{k_{2}}{r}+\frac{k_{3}}{r}\right)} \\
& \times \exp \left[-s(1+p \gamma) \frac{x}{W}\right] \exp \left(-k_{1} g \frac{x}{W}\right) \\
& \quad \times \exp \left[\left(k_{1} \theta \frac{k_{2}}{r} \frac{x}{W}\right) /\left(s+\frac{k_{2}}{r}+\frac{k_{3}}{r}\right)\right] \\
= & k_{1} \bar{U}(0, s) \exp \left[-s(1+p \gamma) \frac{x}{W}\right] \\
& \quad \times \exp \left(-k_{1} \theta \frac{x}{W}\right) \sum_{n=0}^{\infty} \frac{\left(k_{1} \theta \frac{k_{2}}{r} \frac{x}{W}\right)^{n}}{\left(s+\frac{k_{2}}{r}+\frac{k_{3}}{r}\right)^{n} n !} .
\end{aligned}
$$


From which, for an impulse input,

$$
\begin{array}{r}
z(x, t)=k_{1} \frac{q_{0}}{F_{s}} \exp \left[-\left(\frac{k_{2}}{r}+\frac{k_{3}}{r}\right)\left(t-[1+p \gamma] \frac{x}{W}\right)\right] \\
\times \exp \left(-k_{1} \theta \frac{x}{W}\right) \sum_{n=0}^{\infty} \frac{\left(k_{1} \theta \frac{k_{2}}{r} \frac{x}{W}\right)^{n}\left(t-[1+p \gamma] \frac{x}{W}\right)^{n}}{n ! n !} \\
=k_{1} \frac{q_{0}}{F_{s}} \exp \left[-\left(\frac{k_{2}}{r}+\frac{k_{3}}{r}\right)\left(t-[1+p \gamma] \frac{x}{W}\right)\right] \\
\times \exp \left(-k_{1} \theta \frac{x}{W}\right) I_{0}\left(2 \sqrt{k_{1} \theta \frac{k_{2}}{r}} \frac{x}{W}\left(t-[1+p \gamma] \frac{x}{W}\right)\right) \\
\quad \times S\left(t-[1+p \gamma] \frac{x}{W}\right), \quad(8 B)
\end{array}
$$

where $I_{0}(z)$ is a zero order modified Bessel function with argument $z$. The intracellular profile is damped by the term $\exp \left(-k_{3}\left[t-(1+p \gamma) \frac{x}{W}\right]\right)$, when $k_{3}$ assumes finite values. Early in time the effect of this damping factor is not large, for small values of $k_{3}$; but later in time the effect is proportionately much larger.

In these experiments the labeled galactose has been introduced as a tracer input. At the same time the serum galactose level has been set by use of a prolonged intravenous infusion. If we assume that the serum galactose level has become steady, then it is of interest to examine the expressions for the corresponding steady-state sinusoidal and intracellular concentration profiles, as a function of sinusoidal length, since these represent the background against which tracer exchange is occurring. In each case the expression which we wish is the response to a steady infusion [i.e., a step input, for which $\left.\bar{U}(0, s)=\left(q_{0} / F_{0}\right)(1 / s)\right]$; and its form at long time $(t \rightarrow \infty)$. For the sinusoidal concentration we find

$$
u(x, \infty)=\frac{q_{0}}{F_{s}} \exp \left[-\left(k_{1} \theta \frac{k_{3}}{r} \frac{x}{W}\right) /\left(\frac{k_{2}}{r}+\frac{k_{3}}{r}\right)\right] ;
$$

and for the intracellular concentration,

$$
\begin{aligned}
z(x, \infty)= & \frac{k_{1}}{\left[\left(k_{2} / r\right)+\left(k_{3} / r\right)\right]} \frac{q_{0}}{F_{s}} \\
& \quad \times \exp \left[-\left(k_{1} \theta \frac{k_{3}}{r} \frac{x}{W}\right) /\left(\frac{k_{2}}{r}+\frac{k_{3}}{r}\right)\right] .
\end{aligned}
$$

Both profiles decline exponentially. The intracellular profile is proportionately smaller, by the factor $\left(k_{1} /\left[\frac{k_{2}}{r}+\frac{k_{3}}{r}\right)\right]$. We have illustrated in Fig. 11 the manner in which the interrelations between the transport coefficients affect these profiles. As $k_{1}$ increases, the stepdown in concentration across the cell membrane becomes proportionately less, for a given $k_{3}$.

This description of the lengthwise change in intracellular concentration of a substance undergoing metabolic utilization also has a more general connotation. Many substances of

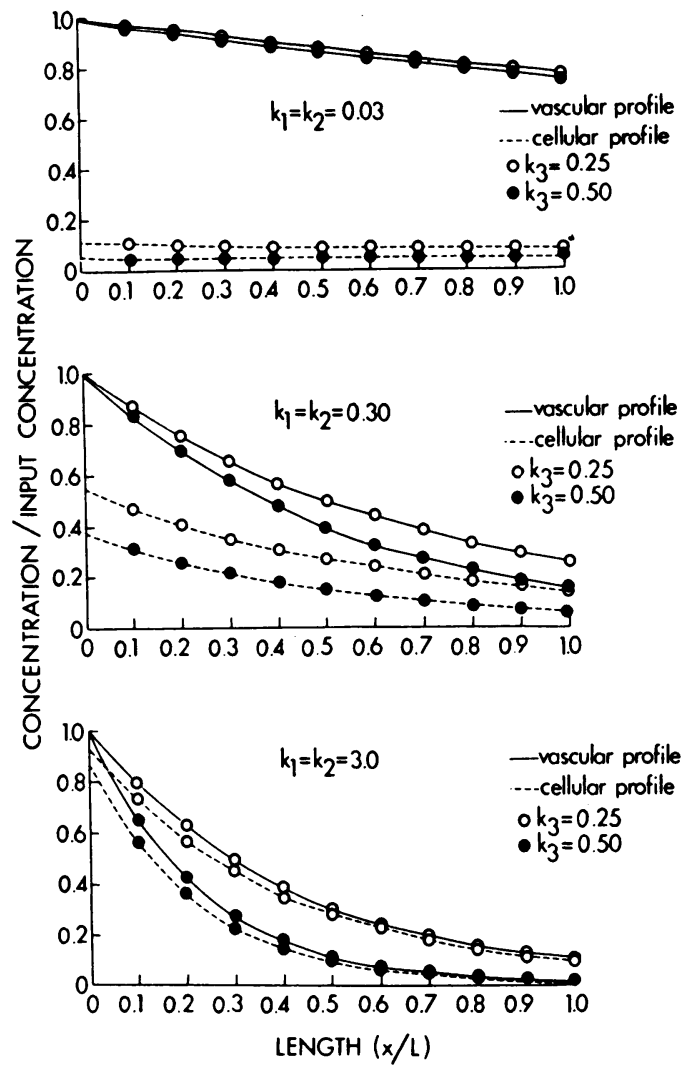

Figure 11 Steady-state intracellular and vascular concentration profiles along the sinusoidal length. These are what would be expected as a result of a long prolonged steady infusion. The vascular profiles are displayed as solid lines; the cellular profiles, as dashed lines. The cellular partition coefficient $r$ is anticipated to be 1.0 ; and the system is defined as equilibrative (i.e., $k_{1}=k_{2}$ ). The values of the parameters $\gamma$ and $\theta$ are 1.0 and 10.0. As $k_{1}$ increases, the corresponding profiles approximate one another.

metabolic importance are present in the blood in relatively unchanging concentration (e.g., oxygen and some substrates). Their metabolic utilization would be expected to result in a concentration profile leading to those circumstances which produce the now well-documented histological zonation within a liver lobule.

Outflow response from the whole liver. We will continue to use the same simplifying assumptions as we have used in the past (5), that the large vessel transit times are uniform, and that the distribution of outflow arrival times of the vascular reference substance occurs chiefly as the result of a distribution of sinusoidal transit times. If we define $Q(t)=$ the quantity of material emerging at the outflow per unit time; $F=$ the total flow through the system; $C(t)=Q(t) / F$, the concentration of material appearing at the outflow; $L=$ the common sinusoidal length (a consequence of the regular quasicrystalline structure of the liver); $n\left(\frac{L}{W_{s}}\right) d\left(\frac{L}{W_{s}}\right)=$ the proportion of sinusoids with transit times from $\left(\frac{L}{W_{s}}\right)$ to $\left(\frac{L}{W_{s}}\right)+d\left(\frac{L}{W_{s}}\right)$, arising from the distribution of sinusoidal velocities; and $q A W_{s} / F=q F_{s} / F=$ the proportion of $q$, the total amount of

Uptake of Galactose By the Intact Liver 
material injected, which enters a sinusoid with the flow $F_{s}$; then

$C(t)=\frac{1}{F} \int_{\left(L / W_{s}\right)_{\min }}^{L / W_{s}} \frac{q F_{8}{ }^{2}}{q_{0} F} u\left(L, t-t_{0}\right) n\left(\frac{L}{W_{8}}\right) d\left(\frac{L}{W_{s}}\right)$,

where $\left(\frac{L}{W_{s}}\right)_{\min }$ is the minimal sinusoidal transit time. Now, for each of the substances which we have used in this experiment, the appropriate "single sinusoidal effect" must be substituted. For convenience let $\tau^{\prime}=t=t_{0}$. Then we find

$$
C\left(\tau^{\prime}\right)_{\mathrm{RBC}}=\frac{1}{F} \frac{q F_{s}}{F} n\left(\tau^{\prime}\right)
$$

for the vascular reference substance;

$$
C\left(\tau^{\prime}\right)_{e c}=\frac{C\left(\tau^{\prime} /[1+p \gamma]\right)_{\mathrm{RBC}}}{(1+p \gamma)}
$$

for the extravascular reference substance; and

$$
\begin{aligned}
& C\left(\tau^{\prime}\right)_{\text {sequ }}=\frac{1}{F} \int_{\left(L / W_{s}\right)_{\min }}^{L / W_{s}} \frac{q F_{s}}{F} \\
& \times \exp \left(-k_{1} \theta \tau^{\prime}\right) \delta\left(\tau^{\prime}-[1+p \gamma] \frac{L}{W_{s}}\right) n\left(\frac{L}{W_{s}}\right) d\left(\frac{L}{W_{s}}\right) \\
& +\frac{1}{F} \int_{\left(I_{1} / W_{s}\right)_{\min }}^{L / W_{s}} \frac{q F_{s}}{F} \exp \left(-k_{1} \theta \frac{L}{W_{s}}\right) \\
& \times \exp \left[-\left(\frac{k_{2}}{r}+\frac{k_{3}}{r}\right)\left(\tau^{\prime}-[1+p \gamma] \frac{L}{W_{s}}\right)\right] \times \\
& \sum_{m=1}^{\infty} \frac{\left(k_{1} \theta \frac{k_{2}}{r} \frac{L}{W_{s}}\right)^{m}\left(\tau^{\prime}-[1+p \gamma] \frac{L}{W_{s}}\right)^{m-1} n\left(\frac{L}{W_{s}}\right) d\left(\frac{L}{W_{s}}\right)}{m !(m-1) !} \\
& =\exp \left(-k_{1} \theta \tau^{\prime}\right) C\left(\tau^{\prime}\right)_{e c}+\exp \left[-\left(\frac{k_{2}}{r}+\frac{k_{3}}{r}\right) \tau^{\prime}\right] \\
& \times \int_{\left(L / W_{s}\right)_{\min }}^{L / W_{s}} \exp \left\{-\left(k_{1} \theta-\left[\frac{k_{2}}{r}+\frac{k_{3}}{r}\right]\right.\right. \\
& \left.\times[1+p \gamma]) \frac{L}{W_{8}}\right\} C\left(\tau^{\prime}\right)_{\mathrm{RBC}} \\
& \times \sum_{m=1}^{\infty} \frac{\left(k_{1} \theta \frac{k_{2}}{r} \frac{L}{W_{s}}\right)^{m}\left(\tau^{\prime}-[1+p \gamma] \frac{L}{W_{s}}\right)^{m-1} d\left(\frac{L}{W_{s}}\right)}{m !(m-1) !}
\end{aligned}
$$

for the substance which is both transported and sequestered. The outflow response for the whole liver consists of two parts, corresponding to the two parts of the single sinusoidal response: throughput material, which sweeps past the liver cells but never enters these cells; and a second term, material which has entered the cells but has escaped the sequestering process, and has later emerged at the outflow.

\section{ACKNOWLEDGMENTS}

We wish to express our appreciation to Miss Susan Aynsley, for her expert technical assistance; and to Miss Margaret Mulherin, for typing this manuscript, with patience and care.

We are indebted to the Medical Research Council of Canada and to the Quebec Heart Foundation for their generous financial support.

\section{REFERENCES}

1. Tygstrup, N., and K. Winkler. 1954. Kinetics of galactose elimination. Acta Physiol. Scand. 32: 354.

2. Tygstrup, N., and K. Winkler. 1958. Galactose blood clearance as a measure of hepatic blood flow. Clin. Sci. $17: 1$.

3. Kalckar, H. M., E. P. Anderson, and K. J. Isselbacher. 1956. Galactosemia, a congenital defect in a nucleotide transferase. Biochim. Biophys. Acta. 20: 262.

4. Isselbacher, K. J., E. P. Anderson, K. Kurahashi, and H. M. Kalckar. 1956. Congenital galactosemia : a single enzymatic block in galactose metabolism. Science (Wash. D. C.). 123: 165 .

5. Goresky, C. A., G. G. Bach, and B. E. Nadeau. 1973. On the uptake of materials by the intact liver. Concentrative transport of rubidium-86. J. Clin. Invest. 52: 975.

6. Chinard, F. P., G. J. Vosburgh, and T. Enns. 1955. Transcapillary exchange of water and other substances in certain organs of the dog. Am. J. Physiol. 183: 221.

7. Goresky, C. A. 1963. A linear method for determining liver sinusoidal and extravascular volumes. $\mathrm{Am} . \mathrm{J}$. Physiol. 204 : 626.

8. Gary-Bobo, C. M., and A. K. Solomon. 1968. Properties of hemoglobin solutions in red cells. J. Gen. Physiol. 52: 825 .

9. Hamilton, W. F., J. W. Moore, J. M. Kinsman, and R. G. Spurling. 1928. Simultaneous determination of the greater and lesser circulation time, of the main velocity through the lungs, of the cardiac output, and an approximation of the amount of blood circulating in the heart and lungs. Am. J. Physiol. 84: 338.

10. Laris, P. C. 1958. Permeability and utilization of glucose in mammalian erythrocytes. J. Cell. Comp. Physiol. 51: 273.

11. Eadie, G. S. 1952. On the evaluation of the constants $V_{m}$ and $K_{m}$ in enzyme reactions. Science (Wash. D. C.). 116: 688.

12. Silverman, M., and C. A. Goresky. 1965. A unified kinetic hypothesis of carrier mediated transport: its applications. Biophys. J. $5: 487$.

13. Hetenyi, G., Jr., and D. Studney. 1967. The distribution of glucose and methylglucose between the liver and plasma in normal and insulin injected rats. Experientia (Basel). 23: 219.

14. Ballard, F. J. 1966. Kinetic studies with liver galactokinase. Biochem. J. 101 : 70.

15. Goresky, C. A., and M. Silverman. 1964. Effect of correction of catheter distortion on calculated liver sinusoidal volumes. Am. J. Physiol. 207: 883 .

16. Hetenyi, G., Jr., K. H. Norwich, D. R. Studney, and J. D. Hall. 1969. The exchange of glucose across the liver cell membrane. Can. J. Physiol. Pharmacol. 47: 361. 
17. Cuatrecases, P., and S. Segal. 1965. Mammalian galactokinase. J. Biol. Chem. 240: 2382.

18. Goresky, C. A. 1970. The interstitial space in the liver: its partitioning effects. In Capillary Permeability. C. Crone and N. A. Lassen, editors. Munksgaard, Copenhagen. 415.

19. Goresky, C. A. 1972. The diffusional problems underlying the distribution of water in well perfused organs: their general implications. In Oxygen Transport and Tissue. M. Kessler, editor. University Park Press, Baltimore, Md. In press.

20. Crone, C. 1971. The blood-brain barrier: facts and questions. In Ion Homeostasis of the Brain. B. K. Siesjo and S. C. Sorenson, editors. Academic Press, Inc., New York. 52.

21. Malone, J. I., H. J. Wells, and S. Segal. 1972. Galactose toxicity in the chick: hyperosmolality or depressed brain energy reserves. Science (Wash. D. C.). 176:816.

22. Levi, A. J., Z. Gaitmaitan, and I. M. Arias. 1969. Two hepatic cytoplasmic protein fractions, $Y$ and $Z$, and their possible role in the hepatic uptake of bilirubin, sulfobromophthalein, and other organic anions. J. Clin. Invest. $48: 2156$.
23. Goresky, C. A. 1964. Initial distribution and rate of uptake of sulfobromophthalein in the liver. Am. J. Physiol. 207: 13.

24. Reyes, H., A. J. Levi, Z. Gaitmaitan, and I. M. Arias. 1969. Organic anion-binding protein in rat liver: drug induction and its physiologic consequence. Proc. Natl. Acad. Sci. $64: 168$.

25. Ziegler, W. H., and C. A. Goresky. 1971. Kinetics of rubidium uptake in the working dog heart. Circ. Res. 29: 208.

26. Ziegler, W. H., and C. A. Goresky. 1971. Transcapillary exchange in the working left ventricle of the dog. Circ. Res. 29: 181.

27. Renkin, E. M. 1959. Transport of potassium-42 from blood to tissue in isolated mammalian skeletal muscles. Am. J. Physiol. $197: 1205$.

28. Crone, C. 1963. The permeability of capillaries in various organs as determined by use of the "indicator diffusion" method. Acta Physiol. Scand. 64 : 407.

29. Perl, W., R. M. Effros, and F. P. Chinard. 1969. Indicator equivalence theorem for input rates and regional masses in multi-inlet steady-state systems with partially labeled input. J. Theor. Biol. 25: 297. 